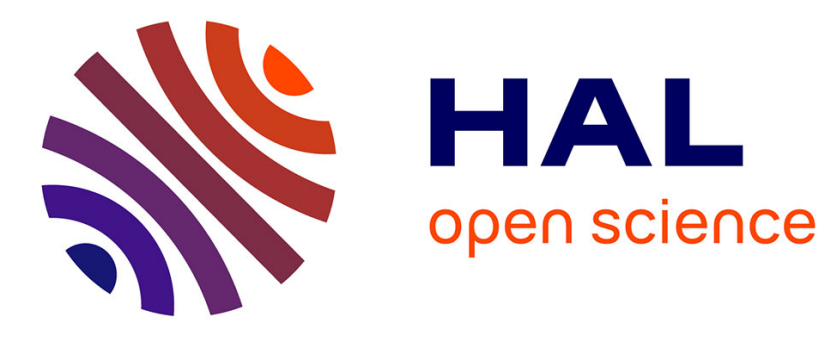

\title{
Self-associated three-dimensional cones
}

Roland Hildebrand

\section{To cite this version:}

Roland Hildebrand. Self-associated three-dimensional cones. Beiträge zur Algebra und Geometrie / Contributions to Algebra and Geometry, 2022, 63, pp.867-906. 10.1007/s13366-021-00599-8 . hal$01900821 \mathrm{v} 2$

\section{HAL Id: hal-01900821 \\ https://hal.science/hal-01900821v2}

Submitted on 23 Oct 2020

HAL is a multi-disciplinary open access archive for the deposit and dissemination of scientific research documents, whether they are published or not. The documents may come from teaching and research institutions in France or abroad, or from public or private research centers.
L'archive ouverte pluridisciplinaire HAL, est destinée au dépôt et à la diffusion de documents scientifiques de niveau recherche, publiés ou non, émanant des établissements d'enseignement et de recherche français ou étrangers, des laboratoires publics ou privés. 


\title{
Self-associated three-dimensional cones
}

\author{
Roland Hildebrand *
}

January 3,2020

\begin{abstract}
For every proper convex cone $K \subset \mathbb{R}^{3}$ there exists a unique complete hyperbolic affine 2-sphere with mean curvature -1 which is asymptotic to the boundary of the cone. Two cones are associated if the corresponding affine spheres can be mapped to each other by an orientation-preserving isometry. This equivalence relation is generated by the groups $S L(3, \mathbb{R})$ and $S^{1}$, where the former acts by linear transformations of the ambient space, and the latter by multiplication of the cubic holomorphic differential of the affine sphere by unimodular complex constants. The group $S^{1}$ generalizes conic duality, which acts by multiplication of the cubic differential by -1 . We call a cone self-associated if it is linearly isomorphic to all its associated cones, in which case the action of $S^{1}$ induces (nonlinear) isometries of the corresponding affine sphere. We give a complete classification of the self-associated cones and compute isothermal parametrizations of the corresponding affine spheres. The solutions can be expressed in terms of degenerate Painlevé III transcendents. The boundaries of generic self-associated cones can be represented as conic hulls of vector-valued solutions of a certain third-order linear ordinary differential equation with periodic coefficients, but there exist also cones with polyhedral boundary parts.
\end{abstract}

\section{Introduction}

In this work we classify the self-associated convex cones in $\mathbb{R}^{3}$, by which we mean those cones that are linearly isomorphic to all its associated cones. The notion of associated cones has been introduced in the paper [23] of Z. Lin and E. Wang and is by virtue of the Calabi theorem derived from the notion of associated families of affine spheres [32]. The results in this paper in spirit partly resemble the work [13] of Dumas and Wolf, which were the first to establish explicit non-trivial relations between affine spheres and their asymptotic cones.

Self-associated cones are akin to semi-homogeneous cones, which have been introduced in [15] and whose corresponding affine spheres have been explicitly computed in [23]. While the affine spheres of the latter possess a continuous isometry group which is generated by linear automorphisms of the cone, the affine spheres of the former have isometries which are of a nonlinear nature and correspond to a continuous symmetry of the cone generalizing the duality symmetry of self-dual cones.

\subsection{Background}

A proper, or regular, convex cone $K \subset \mathbb{R}^{n}$ is a closed convex cone with non-empty interior and containing no line. In the sequel we shall speak of cones for brevity, meaning always proper convex cones. The Calabi conjecture on affine spheres [7], proven by the efforts of several authors, states that for every cone there exists a unique complete hyperbolic affine sphere with mean curvature -1 which is asymptotic to the boundary of the cone $[8,31]$, and conversely, every complete hyperbolic affine sphere is asymptotic to the boundary of a cone $[9,19,20]$. These affine spheres are equipped with a complete non-positively curved Riemannian metric, the affine metric $h$, and a totally symmetric trace-less $(0,3)$-tensor, the cubic form $C$. The two objects are equivariant under the action of the group $S L(n, \mathbb{R})$ of unimodular transformations. Moreover, the affine sphere and the corresponding cone $K$ can be reconstructed from $(h, C)$ up to a unimodular transformation of the ambient space $\mathbb{R}^{n}$. For more on affine spheres see, e.g., [28], for a survey on the Calabi conjecture see [21, Chapter 2].

\footnotetext{
*Univ. Grenoble Alpes, CNRS, Grenoble INP, LJK, 38000 Grenoble, France (roland.hildebrand@univ-grenoble-alpes.fr).
} 
Complete hyperbolic affine 2-spheres are non-compact simply connected Riemann surfaces, and their affine metrics have the form $h=e^{u}|d z|^{2}$ in an isothermal complex coordinate $z=x+i y \in M, M \subset \mathbb{C}$ being a simply connected domain and $u: M \rightarrow \mathbb{R}$ an analytic conformal factor. The cubic form can be represented as $C=2 \operatorname{Re}\left(U(z) d z^{3}\right)$, with $U: M \rightarrow \mathbb{C}$ a holomorphic function, the so-called cubic differential, which satisfies the compatibility condition [33]

$$
e^{u}=\frac{1}{2} \Delta u+2|U|^{2} e^{-2 u}
$$

also called Wang's equation. Here $\Delta u=u_{x x}+u_{y y}=4 u_{z \bar{z}}$ is the Laplacian of $u$. In the sequel, when we speak of solutions $(u, U)$ of $(1)$, we always assume that the metric $h$ is complete and $U$ is holomorphic.

The affine sphere is given by an embedding $f: M \rightarrow \mathbb{R}^{3}$, which is determined by the solution $(u, U)$ up to a unimodular transformation of the ambient space $\mathbb{R}^{3}$. We shall speak of the solution $(u, U)$ of Wang's equation (1) as corresponding to the affine sphere $f$ or the convex cone $K$. In [33] C. Wang deduced the moving frame equations whose integration allows to reconstruct the affine sphere $f$ from a given solution $(u, U)$. Define the $3 \times 3$ real matrix $F=\left(e^{-u / 2} f_{x}, e^{-u / 2} f_{y}, f\right)$. The first two columns of $F$ form an $h$-orthonormal basis of the tangent space to the embedding, while the third column is the position vector of the embedding. The condition that the mean curvature of the affine sphere equals -1 is equivalent to unimodularity of $F$. From the structure equations [32]

$$
f_{z z}=u_{z} f_{z}-U e^{-u} f_{\bar{z}}, \quad f_{z \bar{z}}=\frac{1}{2} e^{u} f, \quad f_{\bar{z} \bar{z}}=-\bar{U} e^{-u} f_{z}+u_{\bar{z}} f_{\bar{z}}
$$

we obtain the frame equations

$$
\begin{aligned}
& F_{x}=F\left(\begin{array}{ccc}
-e^{-u} \operatorname{Re} U & \frac{u_{y}}{2}+e^{-u} \operatorname{Im} U & e^{u / 2} \\
-\frac{u_{y}}{2}+e^{-u} \operatorname{Im} U & e^{-u} \operatorname{Re} U & 0 \\
e^{u / 2} & 0 & 0
\end{array}\right), \\
& F_{y}=F\left(\begin{array}{ccc}
e^{-u} \operatorname{Im} U & -\frac{u_{x}}{2}+e^{-u} \operatorname{Re} U & 0 \\
\frac{u_{x}}{2}+e^{-u} \operatorname{Re} U & -e^{-u} \operatorname{Im} U & e^{u / 2} \\
0 & e^{u / 2} & 0
\end{array}\right) .
\end{aligned}
$$

Choosing an arbitrary point $z_{0}=x_{0}+i y_{0} \in M$ and an arbitrary initial value $F\left(z_{0}\right)=F_{0} \in S L(3, \mathbb{R})$, we can recover the embedding $f$ from the third column of the matrix-valued function $F(z)$ by integrating (2).

The isothermal coordinate system is not unique, but defined up to conformal isomorphisms of the domain $M$. Let $\tilde{M} \subset \mathbb{C}$ be the pre-image of the domain $M$ under the biholomorphic map $b: w \mapsto z$, and consider the embedding $f \circ b: \tilde{M} \rightarrow \mathbb{R}^{3}$. Then the embeddings $f \circ b$ and $f$ define the same affine sphere, considered as a surface in $\mathbb{R}^{3}$, but the parametrization is different. Let $(\tilde{u}, \tilde{U})$ be the solution of (1) corresponding to $f \circ b$. Invariance of the pair $(h, C)$ then yields the transformation law

$$
\tilde{U}(w)=U(z)\left(\frac{d z}{d w}\right)^{3}, \quad \tilde{u}(w)=u(z)+2 \log \left|\frac{d z}{d w}\right| .
$$

The described relation between cubic differentials on domains in the complex plane and 3-dimensional cones has been made more concrete in different cases [33, 26, 18, 27, 1, 2]. Dumas and Wolf [13] have shown that for a non-zero polynomial cubic differential $U$ on the complex plane $\mathbb{C}$ there exists a unique conformal metric $h$ on $\mathbb{C}$ which is compatible with $U$. The pair $(u, U)$ gives rise to an affine sphere which is asymptotic to the boundary of a polyhedral cone in $\mathbb{R}^{3}$, with the degree of the polynomial being equal to the number of extreme rays of the cone less 3 . On the other hand, an affine sphere which is asymptotic to the boundary of a polyhedral cone is conformally equivalent to $\mathbb{C}$ and the corresponding cubic differential is polynomial.

In [32] Simon and Wang studied the compatibility relations between the affine metric $h=e^{u}|d z|^{2}$ and the cubic differential $U$ of an affine 2 -sphere with given mean curvature. Any two $u$-compatible cubic differentials $U$ differ by a multiplicative unimodular complex constant $e^{i \varphi}$, and multiplying a $u$-compatible cubic differential by $e^{i \varphi}$ yields another $u$-compatible cubic differential. Therefore, if an affine 2 -sphere with mean curvature -1 , affine metric $h=e^{u}|d z|^{2}$, and cubic differential $U$ is given, then the affine spheres constructed from the pairs $\left(u, e^{i \varphi} U\right)$, where $\varphi$ runs through $[0,2 \pi)$, exhaust all affine 2 -spheres with affine metric $h$ and mean curvature -1 . The orbits of complete hyperbolic affine 2 -spheres with respect to the action of $S L(3, \mathbb{R})$ 
are hence arranged in 1-parametric associated families, on which the circle group $S^{1} \simeq\{c \in \mathbb{C}|| c \mid=1\}$ acts by multiplication of the cubic differential $U$ by the unimodular group element. Affine spheres belonging to orbits in the same family are called associated [32].

By virtue of the Calabi theorem this notion can naturally be extended to cones in $\mathbb{R}^{3}$. The action of the circle group $S^{1}$ on the solutions of (1) induces an action on the set of $S L(3, \mathbb{R})$-orbits of cones, and these $S L(3, \mathbb{R})$-orbits are also arranged in 1-parametric families. Cones belonging to orbits in the same family are called associated [23].

The action of the group $S^{1}$ on a single associated family of $S L(3, \mathbb{R})$-orbits of cones does not need to be faithful. It may well be that two solutions $(u, U),(u, c U)$ of Wang's equation (1) for $c \neq 1$ lead to isomorphic affine spheres or, equivalently, to the same $S L(3, \mathbb{R})$-orbit of cones. In this contribution we study cones $K \subset \mathbb{R}^{3}$ whose $S L(3, \mathbb{R})$-orbit is a fixed point of the action of $S^{1}$.

Definition 1.1. Let $K \subset \mathbb{R}^{3}$ be a cone and let $(u, U)$ be a solution of (1) corresponding to $K$. For $\varphi \in[0,2 \pi)$, let $K_{\varphi} \subset \mathbb{R}^{3}$ be a cone corresponding to the solution $\left(u, e^{i \varphi} U\right)$ of (1). We call the cone $K$ self-associated if for every $\varphi \in[0,2 \pi)$ the cone $K_{\varphi}$ is linearly isomorphic to $K$.

Note that we did not restrict the linear isomorphisms between $K$ and $K_{\varphi}$ to be unimodular, and hence the condition in Definition 1.1 is a priori weaker than the condition that the $S L(3, \mathbb{R})$-orbit of $K$ is a fixed point of the action of $S^{1}$. Later we shall show that these two conditions are actually equivalent.

Explicit results on associated families of cones are scarce. In [23] the associated families have been computed for the semi-homogeneous cones, i.e., for those cones which have a non-trivial continuous group of linear automorphisms. From the results of Dumas and Wolf [13] it follows that any cone associated to a polyhedral cone is also polyhedral with the same number of extreme rays, and that the self-associated polyhedral cones are exactly the cones over the regular $n$-gons.

In [25, Corollary 4.0.4] Loftin observed that multiplying the cubic differential of an affine 2-sphere by -1 leads to the projectively dual affine 2 -sphere. However, if an affine 2-sphere is asymptotic to the boundary of a convex cone $K \subset \mathbb{R}^{3}$, then the projectively dual affine 2-sphere is asymptotic to the boundary of the dual cone $K^{*}=\left\{y \in \mathbb{R}^{3} \mid x^{T} y \geq 0 \forall x \in K\right\}$. Hence $K^{*}$ is always associated to $K$, and its $S L(3, \mathbb{R})$-orbit can be obtained from the $S L(3, \mathbb{R})$-orbit of $K$ by the action of the group element $e^{i \pi} \in S^{1}$.

Any self-associated cone $K$ must hence be self-dual, in the sense that it is linearly isomorphic to its dual $K^{*}$. The property of being self-associated is therefore stronger than self-duality. The subject of the paper is the description and classification of the self-associated cones.

\subsection{Outline}

In this work we make a step towards a better understanding of associated families of 3-dimensional cones. We provide a full classification of self-associated cones and explicitly describe their boundaries as well as the affine spheres which are asymptotic to these boundaries. We now outline the contents of the paper, sketch the strategy of the proofs, and summarize the results.

In Section 2 we express the condition that a cone $K \subset \mathbb{R}^{3}$ is self-associated equivalently by the analytic conditions $(4),(5)$ on the corresponding solution $(u, U)$ of Wang's equation. These conditions state the existence of a Killing vector field $\psi$ on $M$ whose flow of conformal automorphisms of the domain $M$ multiplies the cubic differential by unimodular complex constants.

In Section 3 we classify the solutions $(\psi, U)$ of (4) up to conformal isomorphisms. We distinguish the obtained pairs $(\psi, U)$ by whether the vector field $\psi$ has a zero in the domain $M$ or not. In the first case the zero can be placed at the origin, the domain is an open disc with radius $R \in(0,+\infty]$, the conformal automorphisms are the rotations of the disc, and $U(z)=z^{k}, k \in \mathbb{N}$. In the second case the domain is a vertical strip $(a, b)+i \mathbb{R}$, where $-\infty \leq a<b \leq+\infty$, the automorphisms are the vertical translations, and $U(z)=e^{z}$. We shall refer to these cases as the rotational and the translational case, respectively.

The trivial solution $U \equiv 0$ which exists for every $\psi$ corresponds to the Lorentz cone $L_{3}$.

In Section 4 we find for every pair $(\psi, U)$ a unique solution $u$ of Wang's equation such that the corresponding metric $h$ is complete on $M$ and $\psi$ is a Killing vector field for $h$ (Lemmas 4.3, 4.4, and 4.5). The last property allows to reduce Wang's equation to the degenerate Painlevé III equation (17). 
Other Painlevé III equations play a role in the description of constant or harmonic inverse mean curvature surfaces with radial symmetry [5],[4], Amsler surfaces [6], and in Smyth surfaces and indefinite affine spheres with intersecting straight lines [3].

The existence and uniqueness of the solution $u$ can in most cases be deduced from similar results in [2] and [13]. In the remaining cases it reduces to existence and uniqueness results on solutions of the Painlevé equation with certain boundary conditions. These can be derived using the asymptotic formulas provided in [17], which is accomplished in Section A in the Appendix.

In Section 5 we integrate the frame equations and compute pieces of the boundary of the self-associated cones. In order to capture the asymptotics of the moving frame at the boundary of the domain we shall employ the technique of osculating frames which has been introduced in [13]. We compare the moving frame $F$ with an explicit diverging unimodular matrix $V$ such that the ratio $G=F V^{-1}$ remains finite and has an explicit limit as the argument tends to the boundary. The matrix $V$ is chosen such that the last column of $G$ and a suitably scaled multiple of the position vector $f$ tend to the same non-zero point in the considered limit. This point must be a boundary point of the cone.

The moving frame equations on $F$ translate to similar partial differential equations on $G$ and in general reduce to a linear third-order ordinary differential equation (ODE) with periodic coefficients on the limit point as a function of the line of convergence. In these cases the corresponding boundary piece of $K$ can be described as the conic hull of a vector-valued solution of the ODE.

In the remaining cases the limit point does not continuously depend on the line along which the limit is taken, but rather yields a discrete sequence of points in $\mathbb{R}^{3}$. In these cases the boundary piece consists of planar faces. Exactly this situation has been encountered by Dumas and Wolf in [13].

In Section 6 we assemble the boundary pieces to obtain the whole boundary of the cone. The main tool to accomplish this task are the linear automorphisms $\Sigma, T$ of the cone that are generated by complex conjugation of the domain and by its rotation by an angle $\frac{2 \pi}{k+3}$ or translation by $2 \pi i$, respectively. These symmetries generate a subgroup of automorphisms which is isomorphic to a finite dihedral group in the rotational case (Lemma 6.2) and to the infinite dihedral group in the translational case (Lemma 6.3).

According to the whether the trace of $T$ is smaller, equal, or greater than 3 we distinguish cones of elliptic, parabolic, and hyperbolic type (Definition 6.1). The first arises in the rotational case, the second in the translational case with $a=-\infty$, and the last in the translational case with $a$ finite.

In Section 7 we summarize our classification in Theorems 7.1, 7.2, and 7.3, describing the three types of cones, respectively.

In Section 8 we pose some open questions and suggest directions for further research.

\section{Killing vectors and self-associated cones}

In this section we characterize affine spheres which are asymptotic to the boundary of self-associated cones by the existence of a Killing vector field such that the 1-parametric group of isometries generated by the field non-trivially multiplies the cubic differential by unimodular complex constants. This will be accomplished in two steps. First we show that if all associated cones $K_{\varphi}$ of a cone $K \subset \mathbb{R}^{3}$ lie in the same $G L(3, \mathbb{R})$-orbit as $K$, then they must also lie in the same $S L(3, \mathbb{R})$-orbit. In a second step we show the existence of the Killing vector field.

\subsection{Isomorphisms with negative determinant}

Suppose two cones $K, \tilde{K}$ are linearly isomorphic, but not in the same $S L(3, \mathbb{R})$-orbit. Then there exists a linear map with determinant -1 taking $K$ to $\tilde{K}$. The next result shows how the corresponding solutions of (1) are related to each other.

Lemma 2.1. Let $K \subset \mathbb{R}^{3}$ be a cone and let $A \in G L(3, \mathbb{R})$ be a linear map with determinant -1 . Let $(u, U)$ be the solution of (1) corresponding to $K$, defined on a simply connected domain $M \subset \mathbb{C}$. Then a solution $(\tilde{u}, \tilde{U})$ of (1) corresponding to the cone $A[K]$ is given by $\tilde{u}(z)=u(\bar{z})$ and $\tilde{U}(z)=\overline{U(\bar{z})}$ on the complex conjugate domain $\bar{M}$. 
Proof. The domain $\bar{M}$ is simply connected, $\tilde{U}$ is holomorphic, and the pair $(\tilde{u}, \tilde{U})$ satisfies Wang's equation (1). Moreover, the metric $\tilde{h}=e^{\tilde{u}}|d z|^{2}$ is complete on $\bar{M}$, because complex conjugation is an isometry between $M$ and $\bar{M}$. Hence $(\tilde{u}, \tilde{U})$ is a solution of (1).

Let $F: M \rightarrow S L(3, \mathbb{R})$ be a solution of the frame equations (2) such that the embedding $f: M \rightarrow \mathbb{R}^{3}$ given by the third column of $F$ is asymptotic to $\partial K$. It is not hard to see that equations (2) are invariant under the substitution $(x, y, u, U, f) \mapsto(x,-y, u, \bar{U},-f)$. Note that $-A \in S L(3, \mathbb{R})$. The embedding $\tilde{f}$ : $\bar{M} \rightarrow \mathbb{R}^{3}$ defined by $\tilde{f}(z)=(-A)(-f(\bar{z}))=A f(\bar{z})$ is then asymptotic to $\partial A[K]$ and gives rise to a solution $\tilde{F}: \bar{M} \rightarrow S L(3, \mathbb{R})$ of the frame equations corresponding to $(\tilde{u}, \tilde{U})$. Therefore the solution $(\tilde{u}, \tilde{U})$ corresponds to the cone $A[K]$.

We now show that for a self-associated cone, the isomorphisms between the associated cones can be assumed to have positive determinant.

Lemma 2.2. A cone $K \subset \mathbb{R}^{3}$ is self-associated if and only if all cones which are associated to $K$ are in the $S L(3, \mathbb{R})$-orbit of $K$.

Proof. The "if" direction follows from the definition of self-associated cones. Let us prove the "only if" direction.

Let $K \subset \mathbb{R}^{3}$ be self-associated. Then every associated cone $K_{\varphi}$ is in the $S L(3, \mathbb{R})$-orbit of $K$ or in the $S L(3, \mathbb{R})$-orbit of $-K$. If $K,-K$ are in the same $S L(3, \mathbb{R})$-orbit, then the claim of the lemma follows. Let us therefore suppose that the $S L(3, \mathbb{R})$-orbits of the cones $K,-K$ are distinct. Define the disjoint complementary subsets $S_{+}, S_{-} \subset S^{1}$ such that $e^{i \varphi} \in S_{ \pm}$if $K_{\varphi}$ is in the $S L(3, \mathbb{R})$-orbit of $\pm K$, respectively.

From (3) it follows by multiplication of the first equation with $e^{i \varphi}$ that if two solutions $(u, U),(\tilde{u}, \tilde{U})$ of Wang's equation are conformally equivalent, then for all $\varphi$ the solutions $\left(u, e^{i \varphi} U\right),\left(\tilde{u}, e^{i \varphi} \tilde{U}\right)$ are also conformally equivalent, by the same isomorphism. Therefore if $e^{i \varphi_{1}}, e^{i \varphi_{2}}$ are in the same subset $S_{+}$or $S_{-}$, then $e^{i\left(\varphi_{1}+\varphi\right)}, e^{i\left(\varphi_{2}+\varphi\right)}$ are also in the same subset for every $\varphi$. In particular, 1 and $e^{i \varphi}$ are in the same subset if and only if $e^{-i \varphi}$ and 1 are in the same subset. It follows that $e^{i \varphi}, e^{-i \varphi}$ are in the same subset. But then $e^{2 i \varphi}, 1$ are in the same subset, for every $\varphi$. Since $1 \in S_{+}$, we obtain $S_{-}=\emptyset$. This completes the proof.

\subsection{Existence of the Killing vector field}

We now proceed to the second step, showing that domains with solutions of (1) corresponding to selfassociated cones carry a Killing vector field with the properties claimed at the beginning of Section 2. A Killing vector field by definition generates a group of orientation-preserving isometries of $M$, which are in particular conformal automorphisms. Therefore Killing vector fields are represented by holomorphic functions $\psi$ in any isothermal coordinate.

Lemma 2.3. Let $K \subset \mathbb{R}^{3}$ be a self-associated cone, and let $(u, U)$ be a corresponding solution of Wang's equation (1) on a domain $M \subset \mathbb{C}$. Then there exists a holomorphic function $\psi$ on $M$ satisfying the relations

$$
\begin{aligned}
i U(z)+U^{\prime}(z) \psi(z)+3 U(z) \psi^{\prime}(z) & =0, \\
\operatorname{Re}\left(u^{\prime}(z) \psi(z)+\psi^{\prime}(z)\right) & =0,
\end{aligned}
$$

where the prime denotes the derivative with respect to the complex coordinate $z$.

Proof. Assume the conditions of the lemma.

If $U \equiv 0$, then $\psi(z) \equiv 0$ satisfies the requirements. Let us henceforth assume that $U \not \equiv 0$.

By Lemma 2.2 all solutions $\left(u, e^{i \varphi} U\right)$ of (1) on $M$ are mutually conformally equivalent. Let Aut $M$ be the finite-dimensional Lie group of biholomorphic automorphisms of $M$. For every $e^{i \varphi} \in S^{1}$, let $G_{\varphi} \subset$ Aut $M$ be the set of all conformal isomorphisms taking the solution $\left(u, e^{i \varphi} U\right)$ of (1) to $(u, U)$. In particular, $G_{0}$ is the subgroup leaving $(u, U)$ and hence $\left(u, e^{i \varphi} U\right)$ invariant for every $\varphi$. Since $e^{i \varphi} U \not \equiv e^{i \varphi^{\prime}} U$ and hence $G_{\varphi} \cap G_{\varphi^{\prime}}=\emptyset$ whenever $e^{i \varphi} \neq e^{i \varphi^{\prime}}$, we may define a surjective map $\alpha: G=\bigcup_{e^{i \varphi} \in S^{1}} G_{\varphi} \rightarrow S^{1}$ by sending $G_{\varphi}$ to $e^{i \varphi}$.

It is easy to check that $G, G_{0}$ are closed subgroups in Aut $M$ and hence Lie subgroups, $G_{0}$ is normal in $G$, and $\alpha$ is a group homomorphism with kernel $G_{0}$. Moreover, $G_{0}$ is closed in the subspace topology of $G$, and hence $G_{0}$ is also a Lie subgroup of $G$. 
If a sequence $b_{k}: w \mapsto z_{k}$ of automorphisms $b_{k} \in$ Aut $M$ tends to the identity map, then for every fixed $w \in M$ we have $b_{k}(w) \rightarrow w$ and $b_{k}^{\prime}(w) \rightarrow 1$. Therefore $\alpha$ is continuous by (3). Thus $\alpha$ is a Lie group homomorphism and defines a group isomorphism between $S^{1}$ and the quotient $G / G_{0}$.

Let $\mathfrak{g}, \mathfrak{g}_{0}$ be the Lie algebras of the Lie groups $G, G_{0}$, respectively. Then $\alpha$ defines a Lie algebra homomorphism $\mathfrak{a}: \mathfrak{g} \rightarrow \mathbb{R}$ of $\mathfrak{g}$ into the Lie algebra $\mathbb{R}$ of $S^{1}$, such that $\mathfrak{g}_{0} \subset$ ker $\mathfrak{a}$. For the sake of contradiction, let us assume that ker $\mathfrak{a}=\mathfrak{g}$. Then the connection component of the identity in $G$ is mapped to $1 \in S^{1}$ and is hence a subset of $G_{0}$. It follows that $G_{0}$ is open in $G$, and the quotient topology of $G / G_{0}$ is discrete. However, $G / G_{0}$ is an uncountable set, because $S^{1}$ is uncountable. Hence $G$ is not second-countable, contradicting the property that it is a finite-dimensional Lie group. Thus ker $\mathfrak{a}$ must be a strict Lie subalgebra of $\mathfrak{g}$, and $\mathfrak{a}$ is surjective.

Let $\mathfrak{b} \in \mathfrak{g}$ be such that $\mathfrak{a}(\mathfrak{b})=-1$. Let $\left\{g_{t}\right\}_{t \in \mathbb{R}} \subset G, g_{t}: w \mapsto z_{t}$, be the one-parametric subgroup generated by the Lie algebra element $\mathfrak{b}$. Then $\alpha\left(g_{t}\right)=e^{-i t}$, and from (3) we get

$$
e^{-i t} U(w)=U\left(z_{t}\right)\left(\frac{d z_{t}}{d w}\right)^{3}, \quad u(w)=u\left(z_{t}\right)+2 \log \left|\frac{d z_{t}}{d w}\right|
$$

for every $t \in \mathbb{R}$.

Let $\psi(w)=\left.\frac{d z_{t}(w)}{d t}\right|_{t=0}$ be the velocity field of the flow generated by $\mathfrak{b}$, represented as a complex-valued function on $M$. Since the $g_{t}$ are isometries, the vector field $\psi$ is a Killing vector field and hence a holomorphic function. By virtue of the relations

$$
z_{0}(w) \equiv w,\left.\quad \frac{d z_{t}}{d w}\right|_{t=0} \equiv 1, \quad\left[\frac{d}{d t} \frac{d z_{t}}{d w}\right]_{t=0}=\psi^{\prime}(w)
$$

differentiation of (6) with respect to $t$ at $t=0$ yields

$$
-i U(w)=U^{\prime}(w) \psi(w)+3 U(w) \psi^{\prime}(w), \quad 0=2 \operatorname{Re}\left(u^{\prime}(w) \psi(w)\right)+2 \operatorname{Re} \psi^{\prime}(w) .
$$

The claim of the lemma readily follows.

The existence of a Killing vector field $\psi$ satisfying (4),(5) is not only necessary, but also sufficient for the cone $K$ to be self-associated. We have the following result.

Lemma 2.4. Let $K \subset \mathbb{R}^{3}$ be a cone, and let $(u, U)$ be a solution of Wang's equation (1) corresponding to $K$, defined on a domain $M \subset \mathbb{C}$. Suppose there exists a holomorphic function $\psi$ on $M$ satisfying relations (4),(5). Then the cone $K$ is self-associated.

Proof. Let $\left\{g_{t}\right\}_{t \in \mathbb{R}}$ be the one-parametric family of conformal maps generated by the vector field $\psi$. For every point $w \in M, z_{t}=g_{t}(w)$ is defined at least in some neighbourhood of $t=0$. Integrating (4),(5) along the trajectories of the flow we obtain (6). In particular, the maps $g_{t}$ are isometries, and the speed along the curve $\left(g_{t}(w)\right)_{t}$ is constant for every fixed $w \in M$. By virtue of the completeness of the metric $h$ on $M, g_{t}$ is hence defined for all $t \in \mathbb{R}$ and is actually a conformal automorphism of $M$. Relation (6) shows that $g_{t}$ takes the solution $\left(u, e^{-i t} U\right)$ of $(1)$ to the solution $(u, U)$ for every $t \in \mathbb{R}$. Hence all these solutions correspond to the same $S L(3, \mathbb{R})$-orbit of cones, and $K$ is self-associated.

In this section we expressed the property of a cone $K \subset \mathbb{R}^{3}$ to be self-associated as a set of two analytic conditions on the corresponding solution of Wang's equation (1). Here condition (4) restricts the holomorphic function $U$ defining the cubic form, and condition (5) restricts the conformal factor $u$ defining the affine metric in isothermal coordinates. In the next section we shall classify the holomorphic functions $U$ which satisfy condition (4), and in Section 4 we find the corresponding functions $u$ satisfying (5).

\section{Classification of cubic holomorphic differentials}

In the previous section we derived a necessary and sufficient condition on a solution $(u, U)$ of Wang's equation (1) to correspond to a self-associated cone, namely equations (4),(5). Our strategy to classify the selfassociated cones will consist in finding all solutions of Wang's equation satisfying these conditions and then constructing the corresponding cones by integrating the moving frame equations. 
In this section we consider condition (4) only. We classify all pairs $(\psi, U)$ of holomorphic functions on $M$ satisfying this condition, where $\psi$ is representing a vector field generating a 1-parametric group of biholomorphic automorphisms of $M$. At first we shall single out the following special case, however.

Case 0: $U \equiv 0$. This condition implies that the cubic form $C$ of the affine sphere vanishes identically. The Theorem of Pick and Berwald [28, Theorem 4.5, p. 53] states that this happens if and only if the affine sphere is a quadric. A quadric can be asymptotic to the boundary of a cone in $\mathbb{R}^{3}$ only if this cone is ellipsoidal, i.e., linearly isomorphic to the Lorentz cone

$$
L_{3}=\left\{x=\left(x_{0}, x_{1}, x_{2}\right)^{T} \mid x_{0} \geq \sqrt{x_{1}^{2}+x_{2}^{2}}\right\} .
$$

The affine sphere is then one sheet of a two-sheeted hyperboloid and is isometric to a hyperbolic space form.

In the rest of this section we assume that $U \not \equiv 0$. Then we also have $\psi \not \equiv 0$. The simply connected domains $M \subset \mathbb{C}$ admitting a 1-parametric group of biholomorphic automorphisms have been classified up to conformal isomorphisms and can be reduced to the following five cases [12, Theorem 3.2]:

(a) $M=\mathbb{C}, \psi(z) \equiv \mu$;

(b) $M=\mathbb{C}, \psi(z)=i \mu z$;

(c) $M=\mathbb{D}, \psi(z)=i \mu z$;

(d) $M=\{z \mid \operatorname{Im} z>0\}, \psi(z) \equiv \mu$;

(e) $M=\{z \mid 0<\operatorname{Im} z<\pi\}, \psi(z) \equiv \mu$.

Here $\mu$ is an arbitrary non-zero real constant. The cases (a),(d),(e) correspond to an automorphism group of translations, the cases (b),(c) to an automorphism group of rotations. For each case we first find all solutions $U$ of (4). In a second step we transform the domain by a conformal isomorphism to obtain a standardized form for $U$.

Case T: If the automorphism group consists of translations, the solutions of (4) are given by $U(z)=$ $\gamma e^{-i z / \mu}$, where $\gamma$ is an arbitrary non-zero complex number. After application of the inverse of the conformal isomorphism $w \mapsto z=\alpha w+\beta$, where $\alpha=i \mu, \beta=-\mu\left(\frac{\pi}{2}+i \log \left(\gamma \mu^{3}\right)\right)$, we obtain by (3) the solution $(\psi, U)=\left(-i, e^{w}\right)$.

The applied map transforms the domain $M$ to the complex plane $\mathbb{C}$ in case (a), to the right half-plane $\left\{w \mid\right.$ Re $\left.w>\log \left|\gamma \mu^{3}\right|\right\}$ in case (b) when $\mu>0$, to the left half-plane $\left\{w|\operatorname{Re} w<\log | \gamma \mu^{3} \mid\right\}$ in case (b) when $\mu<0$, to the vertical strip $\left\{w|\log | \gamma \mu^{3}|<\operatorname{Re} w<\log | \gamma \mu^{3} \mid+\frac{\pi}{\mu}\right\}$ in case (c) if $\mu>0$, and to the vertical strip $\left\{w|\log | \gamma \mu^{3}\left|+\frac{\pi}{\mu}<\operatorname{Re} w<\log \right| \gamma \mu^{3} \mid\right\}$ in case (c) when $\mu<0$.

The solution of (4) can hence be brought to the form $(\psi, U)=\left(-i, e^{w}\right)$ on the domain $M_{(a, b)}=\{w \mid a<$ Re $w<b\}$, which is a vertical strip of finite or infinite width, parameterized by $-\infty \leq a<b \leq+\infty$ or equivalently by the set of non-empty open intervals $(a, b) \subset \mathbb{R}$.

Case R: If the automorphism group consists of rotations, the solutions are locally given by $U(z)=$ $\gamma z^{-(3 \mu+1) / \mu}$, where $\gamma$ is an arbitrary non-zero complex number. This solution is defined in a neighbourhood of $z=0$ if and only if $-\frac{3 \mu+1}{\mu}$ is a natural number, i.e., $\mu=-\frac{1}{k+3}$ for some $k \in \mathbb{N}$. After application of the inverse of the conformal isomorphism $w \mapsto z=\gamma^{\mu} w$ we obtain by (3) the solution $(\psi, U)=\left(-\frac{i w}{k+3}, w^{k}\right)$. The applied map transforms the domain $M$ to the complex plane $\mathbb{C}$ in case (b), and to the open disc $B_{R}=\{w|| w \mid<R\}$ of radius $R=|\gamma|^{-\mu} \in(0,+\infty)$ in case (c).

The solution of $(4)$ can therefore be brought to the form $(\psi, U)=\left(-\frac{i w}{k+3}, w^{k}\right)$, where $k \in \mathbb{N}$ is a discrete parameter, on the domain $B_{R}=\{w|| w \mid<R\}$, which is an open disc of radius $R \in(0,+\infty]$.

Thus in the rotational case we obtain a countably infinite number of 1-parametric families of solutions, while in the translational case we obtain a 2-parametric family of solutions.

In this section we classified the cubic differentials $U$ together with their domain $M$ of definition corresponding to self-associated cones, up to conformal isomorphism. In the next section we find the corresponding solutions $u$ of (1). 


\section{Solving Wang's equation}

In the previous section we have classified all solutions $(\psi, U)$ of equation (4), which in the case $U \not \equiv 0$ led to the canonical forms $z^{k}$ and $e^{z}$ for the holomorphic function $U$ on families of domains $M \subset \mathbb{C}$. In this section we consider the corresponding real-valued function $u$, which has to satisfy both condition (5) and Wang's equation (1) and has to yield a complete metric $h=e^{u}|d z|^{2}$ on $M$.

We shall need the following result due to Benoist and Hulin [2, Proposition 6.8].

Lemma 4.1. Let $M \subset \mathbb{C}$ be a domain which is conformally isomorphic to the unit disc $\mathbb{D}$, and let $h_{0}=$ $e^{u_{0}}|d z|^{2}$ be the uniformizing hyperbolic metric on $M$. Let $U$ be a holomorphic function on $M$ such that the cubic differential $U(z) d z^{3}$ is bounded with respect to $h_{0}$. Then Wang's equation has a unique solution $(u, U)$ such that the metric $e^{u}|d z|^{2}$ is complete on $M$ and $\left|u-u_{0}\right|$ is bounded.

For the domain $\mathbb{C}$ we have the following result due to Dumas and Wolf [13, Theorem 5.3].

Lemma 4.2. Let $M=\mathbb{C}$ and let $U: M \rightarrow \mathbb{C}$ be a polynomial. Then Wang's equation has a unique solution $(u, U)$ on $M$ such that the metric $e^{u}|d z|^{2}$ is complete and has non-positive curvature.

We shall consider the Cases $\mathrm{R}$ and $\mathrm{T}$ from Section 3 separately.

For Case $\mathrm{R}$ we have the following result.

Lemma 4.3. Let $R \in(0, \infty], k \in \mathbb{N}$, and let $U(z)=z^{k}$ be defined on the open disc $B_{R}$ with radius $R$. Then there exists a unique solution $(u, U)$ of $(1)$ on $B_{R}$ that simultaneously satisfies (5) with $\psi(z)=-\frac{i z}{k+3}$.

Proof. Let us first show that for finite $R$ the function $U$ satisfies the conditions of Lemma 4.1. The uniformizing hyperbolic metric on $B_{R}$ is given by $h_{0}=e^{u_{0}}|d z|^{2}$, where $u_{0}(z)=\chi_{0}(r)=2 \log \frac{2 R}{R^{2}-r^{2}}$, and $r e^{i \phi}=z$ are polar coordinates on $B_{R}$. Clearly $h_{0}^{-3 / 2}\left|U d z^{3}\right|=\frac{\left(R^{2}-r^{2}\right)^{3} r^{k}}{8 R^{3}}$ is bounded on $B_{R}$.

Hence Lemmas 4.1, 4.2 are applicable in the cases $R<\infty, R=\infty$, respectively. Let $(u, U)$ be the unique solution of (1) with the properties claimed in these Lemmas. Since these properties, as well as equation (1) by virtue of $|U|=r^{k}$, are invariant with respect to rotations of $B_{R}$, the solution $u$ must be rotationally invariant. In other words, $u(z)=\chi(r)$ for some function $\chi:[0, R) \rightarrow \mathbb{R}$ which can be analytically extended to an even function on $(-R, R)$. But rotational invariance of $u$ is equivalent to (5), which proves the existence of the claimed solution.

We still have to exclude that there exists another solution $(u, U)$ of equations (1),(5) which does not satisfy the additional conditions in Lemmas 4.1 or 4.2. Let $u(z)=\chi(r)$ define such a solution.

Consider first the case $R<\infty$. Completeness of the metric $h=e^{u}|d z|^{2}$ on $B_{R}$ implies that the metric $e^{\chi} d r^{2}$ is complete on $(-R, R)$. In particular, we must have $\lim _{r \rightarrow R} \chi(r)=+\infty$. Plugging $u(z)=\chi(r)$ into equation (1), we obtain the second-order ODE

$$
\frac{d^{2} \chi}{d r^{2}}=2 e^{\chi}-\frac{1}{r} \frac{d \chi}{d r}-4 r^{2 k} e^{-2 \chi}
$$

Making the substitution $e^{\chi}=\sqrt{\frac{k+3}{2}} v r^{(k-1) / 2}, t=\sqrt{\frac{32}{(k+3)^{3}}} r^{(k+3) / 2}$ we obtain a solution $v(t)$ of the Painlevé equation (17) from the Appendix. The boundary condition on $\chi(r)$ is then equivalent to $\lim _{t \rightarrow t_{0}} v(t)=+\infty$, where $t_{0}=\sqrt{\frac{32 R^{k+3}}{(k+3)^{3}}}$, and $v(t)$ must have a double pole with expansion (19) at $t=t_{0}$. Plugging this expansion back into $e^{\chi(r)}$, we obtain

$$
e^{\chi-\chi_{0}}=\left(\frac{1}{(R-r)^{2}}+\frac{1}{R(R-r)}+O(1)\right) / \frac{4 R^{2}}{\left(R^{2}-r^{2}\right)^{2}}=1+O(R-r),
$$

and hence $\lim _{r \rightarrow R}\left(\chi(r)-\chi_{0}(r)\right)=0$. It follows that $\left|\chi-\chi_{0}\right|$ is bounded on $(-R, R)$, and therefore $\left|u-u_{0}\right|$ is bounded on $B_{R}$. Hence the additional condition in Lemma 4.1 holds.

Let us now consider the case $R=+\infty$. The solution $(u, U)$ corresponds to a complete hyperbolic affine sphere. By [7, Theorem 5.1] its metric $h$ is non-positively curved, and the additional condition in Lemma 4.2 holds.

Hence there are no other solutions, which completes the proof. 
For any $k \in \mathbb{N}, R \in(0,+\infty]$ the solution $u$ defines $e^{\chi(r)}$ as an analytic function of $r^{2}$ at $r=0$. Hence the corresponding Painlevé transcendent is of the form $v_{s, c}(t)=t^{-(k-1) /(k+3)} \cdot \kappa\left(t^{4 /(k+3)}\right)$, where $\kappa$ is an analytic function in some neighbourhood of the origin satisfying $\kappa(0)>0$. Comparing with the asymptotic expressions in Section A in the Appendix, we see that the parameter $s=s_{k}$ of the solution depends on $k$ only and is given by $\frac{s_{k}-1}{2}=\cos \frac{2 \pi}{k+3}$.

Moreover, for $R=+\infty$ the parameter $c$ must equal zero, because the corresponding solutions $v_{s_{k}, c}$ are pole-free on the whole positive real axis. For finite $R$ we then have $c>0$, and $c \rightarrow+\infty$ as $R \rightarrow 0$.

Let us now consider Case T. We shall treat the cases $b<+\infty$ and $b=+\infty$ separately.

Lemma 4.4. Let $-\infty \leq a<b<+\infty$, and let $U(z)=e^{z}$ be defined on $M=(a, b)+i \mathbb{R}$. Then there exists a unique solution $(u, U)$ of (1) on $M$ that simultaneously satisfies (5) with $\psi(z)=-i$.

Proof. Let us first show that $U$ satisfies the conditions of Lemma 4.1. For finite $a$ and $a=-\infty$ the uniformizing hyperbolic metric on $M$ is given by $h_{0}=e^{u_{0}(z)}|d z|^{2}=e^{\chi_{0}(x)}|d z|^{2}$ with $e^{\chi_{0}}=\left(\frac{\pi}{b-a}\right)^{2} / \cos ^{2}\left(\frac{\pi}{b-a}\left(x-\frac{a+b}{2}\right)\right)$ and $e^{\chi_{0}}=(b-x)^{-2}$, respectively. It follows that $h_{0}^{-3 / 2}\left|U(z) d z^{3}\right|$ equals $\cos ^{3}\left(\frac{\pi}{b-a}\left(x-\frac{a+b}{2}\right)\right) /\left(\frac{\pi}{b-a}\right)^{3} \cdot e^{x}$ and $(b-x)^{3} \cdot e^{x}$, respectively, which in both cases is bounded on $M$.

Let $(u, U)$ be the unique solution of (1) with the properties asserted in Lemma 4.1. Since these properties, as well as equation (1) by virtue of $|U|=e^{x}$, are invariant with respect to vertical translations, the solution $u$ must be of the form $u(z)=\chi(x)$ for some analytic function $\chi:(a, b) \rightarrow \mathbb{R}$. But translational invariance of $u$ is equivalent to (5), which proves the existence of the claimed solution.

Let us exclude the existence of solutions $(u, U)$ of equations (1),(5) which do not satisfy the additional condition in Lemma 4.1. Let $u(z)=\chi(x)$ define such a solution. Completeness of the metric $h=e^{u}|d z|^{2}$ on $M$ is equivalent to completeness of the metric $e^{\chi} d x^{2}$ on $(a, b)$. Plugging $u$ into equation (1), we obtain the second-order ODE

$$
\frac{d^{2} \chi}{d x^{2}}=2 e^{\chi}-4 e^{2 x} e^{-2 \chi} .
$$

Making the substitution $e^{\chi}=\frac{1}{8} v t, t=\sqrt{32} e^{x / 2}$ we obtain a solution $v(t)$ of the Painlevé equation (17).

Consider first the case of finite $a$. We must have $\lim _{x \rightarrow a} \chi(x)=\lim _{x \rightarrow b} \chi(x)=+\infty$. These conditions are equivalent to $\lim _{t \rightarrow t_{a}} v(t)=\lim _{t \rightarrow t_{b}} v(t)=+\infty$, where $t_{a}=\sqrt{32} e^{a / 2}, t_{b}=\sqrt{32} e^{b / 2}$. Hence $v(t)$ must have double poles with expansion (19) at $t=t_{a}$ and $t=t_{b}$. Plugging this expansion back into $e^{\chi(r)}$, we obtain

$$
e^{\chi-\chi_{0}}=\left(\frac{1}{d^{2}}+O(1)\right) / \frac{\left(\frac{\pi}{b-a}\right)^{2}}{\cos ^{2}\left(\frac{\pi}{b-a}\left(x-\frac{a+b}{2}\right)\right)}=1+O\left(d^{2}\right),
$$

where $d$ equals $x-a$ or $b-x$, respectively. Hence $\lim _{x \rightarrow a}\left(\chi(x)-\chi_{0}(x)\right)=\lim _{x \rightarrow b}\left(\chi(x)-\chi_{0}(x)\right)=0$. It follows that $\left|\chi-\chi_{0}\right|$ is bounded on $(a, b)$, and therefore $\left|u-u_{0}\right|$ is bounded on $M$.

Let us now consider the case $a=-\infty$. As in the previous case $v(t)$ must have a double pole with expansion (19) at $t=t_{b}$, which yields

$$
e^{\chi-\chi_{0}}=\left(\frac{1}{(b-x)^{2}}+O(1)\right) /(b-x)^{-2}=1+O\left((b-x)^{2}\right),
$$

and we get again $\lim _{x \rightarrow b}\left(\chi(x)-\chi_{0}(x)\right)=0$. At the left end of the interval $(-\infty, b)$ the completeness condition is equivalent to the divergence of the integral $\int_{-\infty}^{x} e^{\chi(s) / 2} d s=\int_{0}^{t} \sqrt{\frac{v(s)}{2 s}} d s$. Comparing with the asymptotic expressions in Section A we see that this integral diverges if and only if $\lambda=0$ or equivalently $s=3$. Plugging the asymptotic expression for $v_{3, c}(t)$ back into $\chi(x)$, we obtain $e^{\chi}=x^{-2}+o\left(x^{-2}\right)$ as $x \rightarrow-\infty$. It follows that $e^{\chi-\chi_{0}}=1+o(1)$ and therefore $\lim _{x \rightarrow-\infty}\left(\chi(x)-\chi_{0}(x)\right)=0$. Hence $\left|\chi-\chi_{0}\right|$ is bounded on $(a, b)$, and therefore $\left|u-u_{0}\right|$ is bounded on $M$.

Hence the additional condition in Lemma 4.1 holds in both cases, and there are no other solutions $(u, U)$.

Lemma 4.5. Let $-\infty \leq a<+\infty$, and let $U(z)=e^{z}$ be defined on $M=(a,+\infty)+i \mathbb{R}$. Then there exists a unique solution $(u, U)$ of (1) on $M$ that simultaneously satisfies (5) with $\psi(z)=-i$. 
Proof. In this case $U$ does not satisfy the conditions of Lemma 4.1 and this lemma cannot be used to prove the existence of the solution. However, repeating the reasoning in the proof of the previous lemma, equation (5) is equivalent to the invariance of $u$ with respect to vertical translations, Wang's equation (1) is equivalent to (8) and further to (17), and completeness of the metric $h$ on $M$ is equivalent to completeness of the metric $e^{\chi} d x^{2}$ on $(a,+\infty)$ or of $\frac{v}{2 t} d t^{2}$ on $\left(t_{a},+\infty\right)$, where $t_{a}=\sqrt{32} e^{a / 2}$.

By virtue of the asymptotic expressions in Section A, the solution of (17) must be of the form $v_{s, 0}(t)$ in order to be defined in a neighbourhood of $t=+\infty$. Then, however, we have $v(t) \sim t^{1 / 3}$ for $t \rightarrow+\infty$, and the integral over $\sqrt{t^{-1} v(t)} \sim t^{-1 / 3}$ diverges at the right end of the interval $\left(t_{a},+\infty\right)$. In order for the integral to diverge at the left end it is necessary and sufficient for the solution to have a pole at $t_{a}$ for finite $a$ or to be of the form $v_{3, c}(t)$ for $a=-\infty$, as shown in the proof of the previous lemma. Lemma 4.5 then can be reformulated as follows.

Let $t_{a} \geq 0$ be arbitrary. Then there exists a unique $s \in \mathbb{R}$ such that the solution $v_{s, 0}(t)$ of equation (17) is positive on $\left(t_{a},+\infty\right)$, and either $s=3$ if $t_{a}=0$, or $v_{s, 0}$ has a pole at $t_{a}$ if $t_{a}>0$.

In the case $t_{a}=0$ this reduces to the claim that the solution $v_{3,0}$ is positive on $\mathbb{R}_{++}$. Indeed, consider the sequence of solutions $v_{s_{k}, 0}$, where $\frac{s_{k}-1}{2}=\cos \frac{2 \pi}{k+3}$ for $k \in \mathbb{N}$. We have shown above that $v_{s_{k}, 0}(t)$ is positive for all $t>0$, and we hence have $v_{3,0}(t)=\lim _{k \rightarrow+\infty} v_{s_{k}, 0}(t) \in[0,+\infty]$ for all $t>0$ by continuity of the solutions $v_{s, c}$ with respect to the monodromy parameters $s, c$. Let us show that $v_{3,0}$ cannot have zeros or poles.

The first claim follows from Lemma A.1 and positivity of $v_{s_{k}, 0}$. Suppose now that $v_{3,0}$ has a double pole at $t_{0}$. Then there exists a sequence of points $t_{k} \rightarrow t_{0}$ such that $v_{s_{k}, 0}$ has a local maximum at $t_{k}$ for all large enough $k$, and $v_{s_{k}, 0}\left(t_{k}\right) \rightarrow+\infty$. But then it follows from (17) that $v_{s_{k}, 0}^{\prime \prime}\left(t_{k}\right)=\frac{v_{s_{k}, 0}\left(t_{k}\right)^{3}-t_{k}}{t_{k} v_{s_{k}, 0}\left(t_{k}\right)} \rightarrow+\infty$, a contradiction with the maximality condition $v_{s_{k}, 0}^{\prime \prime}\left(t_{k}\right) \leq 0$. Hence the solution $v_{3,0}$ is finite and positive for all $t>0$.

Let us now consider the case $t_{a}>0$. There exists a 1-parametric family $\left(v_{\alpha}\right)_{\alpha \in \mathbb{R}}$ of solutions of equation (17) which have a double pole at $t=t_{a}$ with expansion (19) around $t_{0}=t_{a}$. By the previous lemma, for every $t_{b}>t_{a}$ there exists exactly one solution in this family which is positive on $\left(t_{a}, t_{b}\right)$ and has a double pole at $t=t_{b}$. Let its parameter be $\alpha=\alpha\left(t_{b}\right)$.

The function $t_{b} \mapsto \alpha\left(t_{b}\right)$ is clearly continuous, and by Lemma A.2 strictly decreasing. By Corollary A.3 we cannot have $\alpha\left(t_{b}\right)<0$ for any $t_{b}>t_{a}$. Hence the limit $\alpha^{*}=\lim _{t_{b} \rightarrow+\infty} \alpha\left(t_{b}\right) \geq 0$ exists. For every $t>t_{a}$, the value $v_{\alpha\left(t_{b}\right)}(t)$ is decreasing as a function of $t_{b}$ for $t_{b}>t$ by Lemma A.2. Hence the limit $\lim _{t_{b} \rightarrow+\infty} v_{\alpha\left(t_{b}\right)}(t)=v_{\alpha^{*}}(t)$ is finite and nonnegative, and the function $v_{\alpha^{*}}$ is nonnegative and pole-free on $\left(t_{a},+\infty\right)$. Moreover, it must be positive on this interval, because $v$ and $v^{\prime}$ cannot be simultaneously zero for any solution of (17). Thus $v_{\alpha^{*}}=v_{s, 0}$ for some $s$, which proves the existence of the desired solution. Its uniqueness follows from Corollary A.4.

By Lemma A.1 the parameter $s$ as a function of $t_{a}$ is strictly increasing and runs through the interval $(3,+\infty)$ for $t_{a} \in \mathbb{R}_{++}$.

In this section we have shown that for every choice of the domain $M$ in both Case $\mathrm{R}$ and Case $\mathrm{T}$ described in Section 3 Wang's equation (1) has a unique solution $u$ which satisfies (5) and such that the metric $h=e^{u}|d z|^{2}$ is complete on $M$. We are now ready to obtain the corresponding self-associated cones in the next sections.

\section{$5 \quad$ Integration of the frame equations}

In order to describe the cone $K$ corresponding to a solution $(u, U)$ of Wang's equation we have to integrate the frame equations (2). Employing the technique of osculating frames introduced in [13] we obtain a description of the boundary $\partial K$.

Recall that the affine sphere $f(z)$ which is asymptotic to $\partial K$ is given by the third column of the unimodular frame $F(z)$. In order to capture its asymptotics we compare $F$ to an explicit osculating frame $V(z)$, such that the ratio $G=F V^{-1}$ remains finite at the boundary of the domain $M$. We can then read off $\partial K$ from the limit $G_{0}$ of $G$. Different pieces of the boundary necessitate different osculating frames, and in this section we compute only these individual pieces.

In order to treat Case $\mathrm{R}$ in the same framework as Case $\mathrm{T}$, we apply the coordinate transformation $z \mapsto w=-3 \log (k+3)+(k+3) \log z$ which transforms the cubic differential $U=z^{k}$ into $\tilde{U}=e^{w}$, the solution $\chi$ of (7) into the solution $\tilde{\chi}=\chi-\frac{2 k}{k+3} \log (k+3)+\frac{2 x}{k+3}$ of (8), the independent variable $r$ of (7) into 
the independent variable $x=-3 \log (k+3)+(k+3) \log r$ of $(8)$, and the punctured domain $B_{R} \backslash\{0\}$ into $(-\infty, b)+i(-(k+3) \pi,(k+3) \pi]$ with $b=-3 \log (k+3)+(k+3) \log R \in(-\infty,+\infty]$. It is more convenient, however, to work with the universal cover of $B_{R} \backslash\{0\}$, which is mapped to $(-\infty, b)+i \mathbb{R}$. We then have to keep in mind that the points $(x, y)$ and $(x, y+2(k+3) \pi)$ in this domain correspond to the same point on the affine sphere. In particular, the moving frame satisfies the relation $F(x, y+2(k+3) \pi)=F(x, y)$ for all $x<b, y \in \mathbb{R}$.

In both Cases $\mathrm{R}$ and $\mathrm{T}$ the domain $M$ of definition of the solution $(u, U)$ is now given by $(a, b)+i \mathbb{R}$, where $-\infty \leq a<b \leq+\infty$.

The frame equations (2) take the form

$$
\begin{aligned}
& F_{x}=F\left(\begin{array}{ccc}
-e^{-\chi} e^{x} \cos y & e^{-\chi} e^{x} \sin y & e^{\chi / 2} \\
e^{-\chi} e^{x} \sin y & e^{-\chi} e^{x} \cos y & 0 \\
e^{\chi / 2} & 0 & 0
\end{array}\right)=: F A \\
& F_{y}=F\left(\begin{array}{ccc}
e^{-\chi} e^{x} \sin y & -\frac{\chi^{\prime}}{2}+e^{-\chi} e^{x} \cos y & 0 \\
\frac{\chi^{\prime}}{2}+e^{-\chi} e^{x} \cos y & -e^{-\chi} e^{x} \sin y & e^{\chi / 2} \\
0 & e^{\chi / 2} & 0
\end{array}\right)=: F B .
\end{aligned}
$$

We shall now study the asymptotic behaviour of the frame $F(x, y)$ as $x$ tends to the limiting values $a$ or $b$. We shall treat the cases of finite and infinite limiting values separately, which yields 4 cases in total to consider.

Case $x \rightarrow b=+\infty$ may be treated by the methods developed in [13]. Consider a horizontal strip $S=(a,+\infty)+i\left(y_{0}, y_{0}+3 \pi\right) \subset M$ of width $3 \pi$. Applying the coordinate transformation $z \mapsto w=2^{1 / 6} \cdot 3 \cdot e^{z / 3}$, we map $U=e^{z}$ to $\tilde{U}=\frac{\sqrt{2}}{2}$, the strip $S$ to some half-plane in $\mathbb{C}$ minus possibly a compact disc centered on the origin, the coordinate $x$ to the radius $r=2^{1 / 6} \cdot 3 \cdot e^{x / 3}$, and $u(z)=\chi(x)$ to a radial function $\tilde{u}(w)=\tilde{\chi}(r)=\chi(x)-\frac{1}{3} \log 2-\frac{2 x}{3}$.

Recall that $e^{\chi}=\frac{v_{s, 0} t}{8}$, where $t=\sqrt{32} e^{x / 2}$, and $v_{s, 0}(t)$ is a solution of the Painlevé equation (17). From its asymptotics in Section A we obtain that

$$
e^{\chi} \sim 2^{1 / 3} e^{2 x / 3}+2^{-1 / 2} 3^{-1 / 4} \pi^{-1 / 2} s e^{x / 2} e^{-2^{2 / 3} 3^{3 / 2} e^{x / 3}}
$$

as $x \rightarrow+\infty$ and hence

$$
e^{\tilde{\chi}} \sim 1+2^{-3 / 4} 3^{1 / 4} \pi^{-1 / 2} s r^{-1 / 2} e^{-\sqrt{6} r}
$$

as $r \rightarrow \infty$. This is precisely the asymptotics obtained in Section 5 and needed for the results in Section 6 of [13] to be applicable.

As a consequence, the affine sphere $f(x, y)$ exhibits the following limiting behaviour as $x \rightarrow+\infty$ for fixed $y$. For $\frac{y}{2 \pi}$ not an integer, the limit of the ray through $f(x, y)$ depends only on the integer part of $\frac{y}{2 \pi}$. The limits of two neighboring strips of width $2 \pi$ are distinct. The ray through $f(x, y)$ with $\frac{y}{2 \pi}$ an integer tends to a proper convex combination of the limiting rays of the neighboring strips. The other convex combinations of these limiting rays can be obtained as the limit of the rays through $f(x, y(x))$ along a curved path on $M$ as $x \rightarrow+\infty$.

Thus in this case the cone $K$ has a polyhedral boundary piece, with $k+3$ extreme rays in Case $\mathrm{R}$ and infinitely many extreme rays in Case T. In Case $\mathrm{R}$ this is already the whole boundary, and $K$ is polyhedral. The last assertion also follows from [13, Theorem 6.3].

Case $x \rightarrow a>-\infty$ : The solution $v(t)$ of the Painlevé equation (17) corresponding to $\chi(x)$ has a double pole at $t_{0}=\sqrt{32} e^{a / 2}$ with expansion (19). Plugging this expansion into $e^{\chi}=\frac{v t}{8}$ and writing shorthand $\delta=x-a$, or equivalently, $t-t_{0}=\sqrt{32} e^{a / 2}\left(e^{\delta / 2}-1\right)$, we obtain the expansions

$$
\begin{gathered}
e^{\chi}=\delta^{-2}+\tilde{\alpha}+O\left(\delta^{2}\right), \quad e^{\chi / 2}=\delta^{-1}+\frac{\tilde{\alpha}}{2} \delta+O\left(\delta^{3}\right), \\
e^{-\chi+\delta}=\delta^{2}+\delta^{3}+\left(\frac{1}{2}-\tilde{\alpha}\right) \delta^{4}+\left(\frac{1}{6}-\tilde{\alpha}\right) \delta^{5}+O\left(\delta^{6}\right), \quad \frac{\chi^{\prime}}{2}=-\delta^{-1}+\tilde{\alpha} \delta+O\left(\delta^{3}\right),
\end{gathered}
$$

where $\tilde{\alpha}=\frac{\alpha e^{a / 2}}{\sqrt{2}}-\frac{1}{48}$, and $\alpha$ is the parameter from (19). 
Let us further consider the matrix variable $G=F V^{-1}$, where $V=\left(\begin{array}{ccc}0 & 0 & \delta \\ 0 & 1 & 0 \\ -\delta^{-1} & 0 & \delta^{-1}\end{array}\right)$. Since $V$ is unimodular, we have $G \in S L(3, \mathbb{R})$. The frame equations (9) become

$$
G_{x}=G V A V^{-1}-G V_{x} V^{-1}, \quad G_{y}=G V B V^{-1} .
$$

Inserting the above expansions, we obtain

$$
\begin{aligned}
& G_{x}=G\left(\begin{array}{ccc}
\frac{\tilde{\alpha}}{2} \delta+O\left(\delta^{3}\right) & 0 & -\delta-\frac{\tilde{\alpha}}{2} \delta^{3}+O\left(\delta^{5}\right) \\
e^{a}\left(\delta+\delta^{2}\right) \sin y+O\left(\delta^{3}\right) & e^{a}\left(\delta^{2}+\delta^{3}\right) \cos y+O\left(\delta^{4}\right) & -e^{a}\left(\delta^{3}+\delta^{4}\right) \sin y+O\left(\delta^{5}\right) \\
e^{a}(1+\delta) \cos y+O\left(\delta^{2}\right) & -e^{a}\left(\delta+\delta^{2}\right) \sin y+O\left(\delta^{3}\right) & -\frac{\tilde{\alpha}}{2} \delta-e^{a} \delta^{2} \cos y+O\left(\delta^{3}\right)
\end{array}\right), \\
& 0 \\
& G_{y}=G\left(\begin{array}{ccc}
\frac{3 \tilde{\alpha}}{2}+e^{a} \delta \cos y+O\left(\delta^{2}\right) & -e^{a}\left(\delta^{2}+\delta^{3}\right) \sin y+O\left(\delta^{4}\right) & 1-\tilde{\alpha} \delta^{2}+O\left(\delta^{3}\right) \\
-e^{a}(1+\delta) \sin y+O\left(\delta^{2}\right) & \frac{3 \tilde{\alpha}}{2}-e^{a} \delta \cos y+O\left(\delta^{2}\right) & e^{a}\left(\delta^{2}+\delta^{3}\right) \sin y+O\left(\delta^{4}\right)
\end{array}\right) .
\end{aligned}
$$

Since the limit of the derivatives of $G$ as $\delta \rightarrow 0$ exists and the convergence is uniform in $y$, the function $G(x, y)$ extends analytically to some unimodular matrix-valued function $G_{0}(y)=: G(a, y)$. This function obeys the linear ODE

$$
\frac{d G_{0}}{d y}=G_{0} \cdot\left(\begin{array}{ccc}
0 & 1 & 0 \\
\frac{3 \tilde{\alpha}}{2} & 0 & 1 \\
-e^{a} \sin y & \frac{3 \tilde{\alpha}}{2} & 0
\end{array}\right)
$$

with the $2 \pi$-periodic coefficient matrix being the limit of $G_{y}$ as $\delta \rightarrow 0$.

Denote the third column of $G_{0}$ by $f_{0}$. The vector $f_{0}(y)$ never vanishes and obeys the linear third-order ODE

$$
\frac{d^{3} f_{0}}{d y^{3}}-3 \tilde{\alpha} \cdot \frac{d f_{0}}{d y}+e^{a} \sin y \cdot f_{0}=0
$$

with $2 \pi$-periodic coefficients. We have

$$
\operatorname{det}\left(f_{0}^{\prime \prime}, f_{0}^{\prime}, f_{0}\right)=\operatorname{det}\left[G_{0} \cdot\left(\begin{array}{ccc}
1 & 0 & 0 \\
0 & 1 & 0 \\
\frac{3 \tilde{\alpha}}{2} & 0 & 1
\end{array}\right)\right]=1
$$

Note that the vector $\delta \cdot f(x, y)=G \cdot\left(\delta^{2}, 0,1\right)^{T}$ tends to $f_{0}(y)$ for $x \rightarrow a$. Hence the curve $f_{0}: \mathbb{R} \rightarrow \mathbb{R}^{3}$ lies on the boundary of the cone $K$. It is analytic and generates an injective map from $\mathbb{R}$ into the manifold of boundary rays of $K$, because by virtue of (11) the derivative $\frac{d f_{0}}{d y}$ and the vector $f_{0}$ are linearly independent. The boundary piece of $K$ defined by the conic hull of the curve $f_{0}$ is analytic at every point which is distinct from the tip of the cone.

Case $x \rightarrow b<+\infty$ : Replacing $a$ by $b$ we obtain the same expansions and can perform the same constructions as in the previous case. However, since now $x \rightarrow b$ from the left, we have that $\delta=x-b$ is negative. Therefore we must define $f_{0}(y)$ as minus the third column of the matrix $G_{0}(y)$ in order for it to be the limit of $-\delta \cdot f(x, y)$ as $x \rightarrow b$. The vector-valued function $f_{0}$ is a solution of the linear third-order ODE

$$
\frac{d^{3} f_{0}}{d y^{3}}-3 \tilde{\alpha} \cdot \frac{d f_{0}}{d y}+e^{b} \sin y \cdot f_{0}=0
$$

with $\tilde{\alpha}=\frac{\alpha e^{b / 2}}{\sqrt{2}}-\frac{1}{48}, \alpha$ being the parameter from expansion (19) at $t_{0}=\sqrt{32} e^{b / 2}$. It satisfies the condition

$$
\operatorname{det}\left(f_{0}^{\prime \prime}, f_{0}^{\prime}, f_{0}\right)=-1 \text {. }
$$

The conic hull of the curve $f_{0}(y), y \in \mathbb{R}$, again defines an analytic boundary piece of $K$. In Case $\mathrm{R}$ this curve must be closed with period $2(k+3) \pi$ by virtue of the relation $f(x, y+2(k+3) \pi)=f(x, y)$, and hence its conic hull makes up the whole boundary $\partial K$. In Case $\mathrm{T} f_{0}$ again generates an injective map from $\mathbb{R}$ into the manifold of boundary rays of $K$. 
Case $x \rightarrow a=-\infty$ : From Lemma A.5 we obtain the expansions

$$
\begin{gathered}
e^{\chi}=\frac{1}{(-x+\alpha)^{2}}+O\left(e^{2 x} x^{2}\right), \quad e^{-\chi+x}=(-x+\alpha)^{2} e^{x}+O\left(e^{3 x} x^{6}\right), \\
e^{\chi / 2}=\frac{1}{-x+\alpha}+O\left(e^{2 x} x^{3}\right), \quad \frac{\chi^{\prime}}{2}=\frac{1}{-x+\alpha}+O\left(e^{2 x} x^{3}\right)
\end{gathered}
$$

as $x \rightarrow-\infty$, where $\alpha=\log 2-3 \gamma-\frac{3}{2} c, \gamma$ is the Euler-Mascheroni constant, and $c$ is the parameter in the solution $v_{3, c}(t)$ of the Painlevé equation (17) which determines $\chi(x)$.

Set $\delta=\frac{1}{-x+\alpha}$ and define the matrix-valued function $G=F V^{-1}$ with $V=\left(\begin{array}{ccc}\frac{\delta}{2} & 0 & \frac{\delta}{2} \\ 0 & 1 & 0 \\ -\delta^{-1} & 0 & \delta^{-1}\end{array}\right)$. Again $V$ is unimodular and hence $G \in S L(3, \mathbb{R})$. By virtue of the above expansions and the relation $\frac{d \delta}{d x}=\delta^{2}$ the frame equations become

$$
\begin{aligned}
& G_{x}=G V A V^{-1}-G V_{x} V^{-1}=G\left(\begin{array}{ccc}
O\left(\delta^{-2} e^{x}\right) & O\left(\delta^{-1} e^{x}\right) & O\left(e^{x}\right) \\
O\left(\delta^{-3} e^{x}\right) & O\left(\delta^{-2} e^{x}\right) & O\left(\delta^{-1} e^{x}\right) \\
O\left(\delta^{-4} e^{x}\right) & O\left(\delta^{-3} e^{x}\right) & O\left(\delta^{-2} e^{x}\right)
\end{array}\right) \\
& G_{y}=G V B V^{-1}=G\left(\begin{array}{ccc}
O\left(\delta^{-2} e^{x}\right) & O\left(\delta^{-1} e^{x}\right) & O\left(e^{x}\right) \\
2+O\left(\delta^{-3} e^{x}\right) & O\left(\delta^{-2} e^{x}\right) & O\left(\delta^{-1} e^{x}\right) \\
O\left(\delta^{-4} e^{x}\right) & 2+O\left(\delta^{-3} e^{x}\right) & O\left(\delta^{-2} e^{x}\right)
\end{array}\right)
\end{aligned}
$$

Here the constants in the $O$ terms are uniformly bounded over $y \in \mathbb{R}$. It follows that $G(x, y)$ converges to some function $G_{0}(y)$ as $x \rightarrow-\infty$, and this function obeys

$$
\frac{d G_{0}}{d y}=G_{0} \cdot\left(\begin{array}{lll}
0 & 0 & 0 \\
2 & 0 & 0 \\
0 & 2 & 0
\end{array}\right) \Rightarrow G_{0}(y)=G_{0}(0) \cdot\left(\begin{array}{ccc}
1 & 0 & 0 \\
2 y & 1 & 0 \\
2 y^{2} & 2 y & 1
\end{array}\right)
$$

Let again $f_{0}$ be the last column of $G_{0}$, which in this case is just the last column of $G_{0}(0)$ and hence a fixed vector. The vector $\delta \cdot f(x, y)=G(x, y) \cdot\left(\frac{\delta^{2}}{2}, 0,1\right)^{T}$ tends to $f_{0}$ for $x \rightarrow-\infty$ which hence generates a boundary ray $\rho_{0}$ of the cone $K$.

In this section we described the boundary pieces of $K$ to which the affine sphere $f(x, y)$ is asymptotic as $x \rightarrow a$ or $x \rightarrow b$ for bounded $y$. In the cases $x \rightarrow \pm \infty$ we obtain a polyhedral boundary piece and a single ray, respectively, while for finite limits the boundary piece is the conic hull of a vector-valued solution of ODE $(10),(12)$, respectively. In the next section we assemble these pieces in order to construct the whole cones.

\section{Computing the self-associated cones}

In this section we describe the cones $K$ corresponding to the solutions $(u, U)$ analyzed in the previous sections. The most important tool to assemble the boundary pieces obtained in the previous section will be the automorphism group of $K$.

If the cubic differential $U$ is normalized to $e^{z}$, then the coefficient matrices $A, B$ in the frame equations (9) are invariant with respect to the translation $(x, y) \mapsto(x, y+2 \pi)$. Hence the gradient of the expression $F(x, y+2 \pi) F^{-1}(x, y)$ vanishes, and there exists a unimodular matrix $T$ such that $F(x, y+2 \pi)=T F(x, y)$ for all $x+i y \in M$. In particular, $f(x, y+2 \pi)=T f(x, y)$, and $T$ is a linear automorphism of the cone $K$ to which the surface $f$ is asymptotic. In Case $\mathrm{R}$ we have $F(x, y+2(k+3) \pi)=F(x, y)$, which implies $T^{k+3}=I$.

Let us introduce the diagonal matrix $D=\operatorname{diag}(1,-1,1)$. Then $A(x,-y)=D A(x, y) D, B(x,-y)=$ $-D B(x, y) D$ for all $x+i y \in M$. Hence the gradient of the expression $F(x,-y) D F^{-1}(x, y)$ vanishes, and there exists a matrix $\Sigma$ with determinant equal to -1 such that $F(x,-y)=\Sigma F(x, y) D$ for all $x+i y \in M$. Again we have $f(x,-y)=\Sigma f(x, y)$, and $\Sigma$ is a linear automorphism of $K$. Clearly it satisfies the relation $\Sigma^{2}=I$.

We have the relation $T \Sigma F(x, y)=T F(x,-y) D=F(x,-y+2 \pi) D$ and hence

$$
(T \Sigma)^{2} F(x, y)=F(x,-(-y+2 \pi)+2 \pi) D^{2}=F(x, y) .
$$


It follows that $(T \Sigma)^{2}=I$, and in particular, that $T$ and $T^{-1}$ are conjugated by $\Sigma$. Therefore $T$ must have spectrum $\left\{1, \lambda, \lambda^{-1}\right\}$ with $\lambda$ on the real line or on the unit circle.

Definition 6.1. We shall call the self-associated cone $K$ of

- elliptic type if its automorphism $T$ has spectrum $\left\{1, e^{i \varphi}, e^{-i \varphi}\right\}$ with $\varphi$ not a multiple of $\pi$;

- parabolic type if $T$ has spectrum in $\{-1,+1\}$;

- hyperbolic type if $T$ has spectrum $\left\{1, \lambda, \lambda^{-1}\right\}$ with $|\lambda|>1$.

Besides the automorphisms $T, \Sigma$, the moving frame allows to define an invariant symmetric matrix. Set $J=\operatorname{diag}(1,-1,-1)$. Then $A(x, y-\pi) J+J A^{T}(-y)=0, B(x, y-\pi) J-J B^{T}(-y)=0$. Hence the gradient of the expression $\Omega=F(x, y-\pi) J F^{T}(x,-y)$ vanishes, and this unimodular matrix is constant over the domain $M$. If we replace $y$ by $-y+\pi$ we obtain the transpose of this matrix, which shows that $\Omega$ is symmetric.

We shall now consider the cones corresponding to the different solutions $(u, U)$ studied in the previous sections.

Lemma 6.2. In Case $R$ the subgroup of automorphisms of $K$ generated by $T$ and $\Sigma$ is isomorphic to the dihedral group $D_{k+3}$. The cones $K$ are of elliptic type.

Proof. It is more convenient to pass back to the domain $M=B_{R}$, with the cubic differential given by $U=z^{k}$. Let $F(z)$ be the moving frame of the corresponding affine sphere. Then $F\left(e^{2 \pi i /(k+3)} z\right)=T F(z)$, $F(\bar{z})=\Sigma F(z) D$ for all $z \in M$. In particular, $f\left(e^{2 \pi i /(k+3)} z\right)=T f(z), f(\bar{z})=\Sigma f(z)$.

Hence $f(0) \in \mathbb{R}^{3}$ is an eigenvector of both $T$ and $\Sigma$ with eigenvalue 1 . The complementary invariant subspace is also shared by both $T$ and $\Sigma$ and is given by the tangent space to the surface $f(z)$ at $z=0$. It can naturally be parameterized by the complex variable $z=x+i y \in \mathbb{C}$. By definition $T$ acts on this subspace by rotations $z \mapsto e^{2 \pi i /(k+3)} z$ and $\Sigma$ by reflections $(x, y) \mapsto(x,-y)$. Hence the spectrum of $T$ is given by $\left\{1, e^{2 \pi i /(k+3)}, e^{-2 \pi i /(k+3)}\right\}$ and the claims easily follow.

Let us now consider the cones corresponding to Case T.

Lemma 6.3. In Case $T$ the subgroup of automorphisms of $K$ generated by $T$ and $\Sigma$ is isomorphic to the infinite dihedral group $D_{\infty}$. The spectrum of $T$ is real.

Proof. Suppose for the sake of contradiction that $T$ has a complex eigenvalue $e^{i \varphi}$. Then $T$ is diagonalisable, and the sequence $T^{n}, n \in \mathbb{N}$, accumulates to the identity matrix. But then the sequence of vectors $f(x, y+2 \pi n)$ accumulates to $f(x, y)$, contradicting the fact that a complete hyperbolic affine sphere is an embedding. Hence the spectrum of $T$ is real.

The matrices $F(x, y+2 \pi n), n \in \mathbb{Z}$, are mutually distinct. Hence also $T^{n}$ are mutually distinct. Thus the group generated by $T, \Sigma$ is isomorphic to $D_{\infty}$.

Therefore in Case $\mathrm{T}$ the corresponding cones are of either parabolic or hyperbolic type. We now establish that this depends on whether $a=-\infty$ or $a$ finite.

Lemma 6.4. Let $-\infty<b \leq \infty$ and let $K$ be the cone corresponding to the solution of Wang's equation on $(-\infty, b)+i \mathbb{R}$ in Case $T$. Then $K$ is of parabolic type, and $T$ has spectrum $\{1\}$ with a 1-dimensional eigenspace. The corresponding eigenvector generates a boundary ray $\rho_{0}$ of $K$.

Proof. In the previous section the moving frame was represented as a product $F(x, y)=G(x, y) V(x)$, where $G, V$ are unimodular matrix-valued functions. The relation $F(x, y+2 \pi)=T F(x, y)$ then yields the similar relation $G(x, y+2 \pi)=T G(x, y)$. Passing to the limit $x \rightarrow-\infty$, we obtain the relation $G_{0}(y+2 \pi)=T G_{0}(y)$ and by virtue of (14)

$$
T=G_{0}(0)\left(\begin{array}{ccc}
1 & 0 & 0 \\
2(y+2 \pi) & 1 & 0 \\
2(y+2 \pi)^{2} & 2(y+2 \pi) & 1
\end{array}\right)\left(\begin{array}{ccc}
1 & 0 & 0 \\
2 y & 1 & 0 \\
2 y^{2} & 2 y & 1
\end{array}\right)^{-1} \quad G_{0}(0)^{-1}=G_{0}(0)\left(\begin{array}{ccc}
1 & 0 & 0 \\
4 \pi & 1 & 0 \\
8 \pi^{2} & 4 \pi & 1
\end{array}\right) G_{0}(0)^{-1} .
$$

This shows that the only eigenvalue of $T$ is 1 and it has geometric multiplicity 1 .

Moreover, the eigenvector of $T$ is given by the last column $f_{0}$ of $G_{0}(0)$. By construction of $G_{0}$ this vector lies on the boundary of $K$. 
Corollary 6.5. Under the assumptions of the previous lemma, the boundary of $K$ is given by the closure of the conic hull of a vector-valued solution of ODE (12) tending to $\rho_{0}$ both in the forward and the backward direction in the case $b<+\infty$, and by the closure of an infinite chain of of 2-dimensional faces accumulating in both directions to $\rho_{0}$ in the case $b=+\infty$.

Proof. Let $v \in \mathbb{R}^{3}$ be a non-zero vector. Then the sequence of rays generated by $T^{n} v$ tends to either $\rho_{0}$ or $-\rho_{0}$ as $n \rightarrow \pm \infty$. Clearly if $v$ lies on $\partial K$, this sequence also lies on $\partial K$ and hence tends to $\rho_{0}$.

Consider first the case $b<+\infty$. Recall that $\partial K$ contains an analytic piece given by the conic hull of a vector-valued solution $f_{0}(y)$ of ODE (12). By construction we have $f_{0}(y+2 \pi)=T f_{0}(y)$ for all $y \in \mathbb{R}$. Setting $v=f_{0}\left(y_{0}\right)$ for $y_{0}$ arbitrary, we see that the ray through $f_{0}(y)$ tends to $\rho_{0}$ as $y \rightarrow \pm \infty$.

In the case $b=+\infty$ the boundary of $K$ contains an infinite chain of planar faces on which $T$ acts by construction by a shift. Setting $v$ equal to any non-zero point in this chain yields the desired conclusion.

Lemma 6.6. Let $-\infty<a<b \leq+\infty$ and let $K$ be the cone corresponding to the solution of Wang's equation on $(a, b)+i \mathbb{R}$. Then $K$ is of hyperbolic type, and $T$ has spectrum $\left\{1, \lambda, \lambda^{-1}\right\}$ with $\lambda>1$.

Proof. Let us assume first that $b$ is finite. By the results of the previous section, the boundary $\partial K$ contains two analytic pieces which are the conic hulls of vector-valued solutions $f_{0}^{ \pm}(y)$ of ODEs (12),(10), respectively. The automorphisms $T, \Sigma$ of $K$ act on these solutions by $T f_{0}^{ \pm}(y)=f_{0}^{ \pm}(y+2 \pi), \Sigma f_{0}^{ \pm}(y)=f_{0}^{ \pm}(-y)$.

The rays through $f_{0}^{+}(y)$ tend to some boundary rays $\rho_{ \pm}^{+}$of $K$ as $y \rightarrow \pm \infty$, respectively. In the same way, the rays through $f_{0}^{-}(y)$ tend to boundary rays $\rho_{ \pm}^{-}$. Clearly these limit rays must be generated by eigenvectors of $T$ corresponding to positive eigenvalues. Moreover, $\Sigma\left[\rho_{ \pm}^{ \pm}\right]=\rho_{\mp}^{ \pm}$.

If all these four rays are mutually distinct, then any three of them cannot be coplanar, otherwise at least one of the analytic boundary pieces has to lie in a flat face of $K$, contradicting (11) or (13). It follows that $T$ has an eigenspace with dimension 3 and must equal the identity, leading to a contradiction.

Hence we have that $\rho_{+}^{-}=\rho_{+}^{+}=: \rho_{+}, \rho_{-}^{-}=\rho_{-}^{+}=: \rho_{-}$, because these two relations can hold only simultaneously by virtue of the symmetry $\Sigma$. Moreover, the product of the eigenvalues of $T$ corresponding to $\rho_{ \pm}$ equals 1 , because $\Sigma$ conjugates $T$ to $T^{-1}$.

Now suppose for the sake of contradiction that the eigenvectors of $T$ generating $\rho_{ \pm}$have eigenvalue 1 . Then the whole 2-dimensional subspace spanned by $\rho_{ \pm}$is left fixed by $T$. However, this subspace intersects the interior of the cone $K$, contradicting the fact that the affine sphere $f(x, y)$ does not contain fixed points of $T$. Hence the eigenvalues corresponding to $\rho_{ \pm}$equal $\lambda^{ \pm 1}$ for some $\lambda>1$, proving our claim.

In the case $b=+\infty$ the argument is similar, with the analytic boundary piece generated by the solution $f_{0}^{+}(y)$ replaced by an infinite chain of 2-dimensional faces.

The proof of the lemma shows that the closure of the union of the two boundary pieces makes up already the whole boundary of $K$. For each of the pieces we have a unimodular initial condition to choose when integrating the moving frame. Only one of these conditions can be chosen freely, corresponding to the choice of the representative in the $S L(3, \mathbb{R})$-orbit of the cone. In order to choose the second initial condition correctly without having to compute the solution $v(t)$ of the Painlevé equation we will make use of the automorphisms $T, \Sigma$, and the invariant symmetric matrix $\Omega$ which are common for both boundary pieces.

Consider first the case $b<+\infty$. For any of the solutions $f_{0}(y)$ defining a boundary piece, set $\Phi=$ $\left(f_{0}^{\prime \prime}, f_{0}^{\prime}, f_{0}\right)$. Then $\Phi(y+2 \pi)=T \Phi(y), \Phi(-y)=\Sigma \Phi(y) D$. Note further that

$$
\lim _{\delta \rightarrow 0} V(\delta) J V^{T}(\delta)=\lim _{\delta \rightarrow 0}\left(\begin{array}{ccc}
0 & 0 & \delta \\
0 & 1 & 0 \\
-\delta^{-1} & 0 & \delta^{-1}
\end{array}\right)\left(\begin{array}{ccc}
1 & 0 & 0 \\
0 & -1 & 0 \\
0 & 0 & -1
\end{array}\right)\left(\begin{array}{ccc}
0 & 0 & -\delta^{-1} \\
0 & 1 & 0 \\
\delta & 0 & \delta^{-1}
\end{array}\right)=\left(\begin{array}{ccc}
0 & 0 & -1 \\
0 & -1 & 0 \\
-1 & 0 & 0
\end{array}\right) .
$$

Hence

$$
\begin{aligned}
& \Omega=G(x, y-\pi) V(\delta) J V^{T}(\delta) G^{T}(x,-y)=G_{0}(y-\pi)\left(\begin{array}{ccc}
0 & 0 & -1 \\
0 & -1 & 0 \\
-1 & 0 & 0
\end{array}\right) G_{0}^{T}(-y) \\
& =\Phi(y-\pi)\left(\begin{array}{ccc}
1 & 0 & 0 \\
0 & 1 & 0 \\
-\frac{3 \tilde{\alpha}}{2} & 0 & 1
\end{array}\right)\left(\begin{array}{ccc}
0 & 0 & -1 \\
0 & -1 & 0 \\
-1 & 0 & 0
\end{array}\right)\left(\begin{array}{ccc}
1 & 0 & -\frac{3 \tilde{\alpha}}{2} \\
0 & 1 & 0 \\
0 & 0 & 1
\end{array}\right) \Phi^{T}(-y)=\Phi(y-\pi)\left(\begin{array}{ccc}
0 & 0 & -1 \\
0 & -1 & 0 \\
-1 & 0 & 3 \tilde{\alpha}
\end{array}\right) \Phi^{T}(-y) .
\end{aligned}
$$


Let now $\Phi_{ \pm}(y)$ be the matrices corresponding to the solutions $f_{0}^{ \pm}(y)$ defining the two boundary pieces. Then we have

$$
\begin{gathered}
\Phi_{+}(2 \pi) \Phi_{+}^{-1}(0)=\Phi_{-}(2 \pi) \Phi_{-}^{-1}(0), \Phi_{+}(0) D \Phi_{+}^{-1}(0)=\Phi_{-}(0) D \Phi_{-}^{-1}(0), \\
\Phi_{+}(-\pi)\left(\begin{array}{ccc}
0 & 0 & -1 \\
0 & -1 & 0 \\
-1 & 0 & 3 \tilde{\alpha}_{b}
\end{array}\right) \Phi_{+}^{T}(0)=\Phi_{-}(-\pi)\left(\begin{array}{ccc}
0 & 0 & -1 \\
0 & -1 & 0 \\
-1 & 0 & 3 \tilde{\alpha}_{a}
\end{array}\right) \Phi_{-}^{T}(0),
\end{gathered}
$$

where $\tilde{\alpha}_{a}, \tilde{\alpha}_{b}$ are the constants in ODEs (10),(12), respectively. Together with the conditions $\operatorname{det} \Phi_{ \pm}= \pm 1$ and the condition that the conic hulls of the solutions $f_{0}$ meet at the same rays $\rho_{ \pm}$as $y \rightarrow \pm \infty$, respectively, these relations define $\Phi_{+}$uniquely for given $\Phi_{-}$and vice versa.

Now we consider the case $b=+\infty$. The formulas involving $\Phi_{-}$remain the same, but we have to find a suitable replacement for those involving $\Phi_{+}$. Recall that the ray through $f(x,(2 n-1) \pi), n \in \mathbb{Z}$, tends to an extreme ray $\rho_{n}$ of $K$, and that $T\left[\rho_{n}\right]=\rho_{n+1}, \Sigma\left[\rho_{n}\right]=\rho_{1-n}$ for all $n$. Choose a non-zero point $v_{0} \in \rho_{0}$ and define $v_{n}=T^{n} v_{0} \in \rho_{n}$ for all $n$. By the relation $(T \Sigma)^{2}=I$ we then have $\Sigma v_{n}=v_{1-n}$. Application of the methods in [13, Section 6] leads to the formula $\Omega=|\operatorname{det} H|^{-2 / 3} H\left(\begin{array}{ccc}-1 & 0 & 0 \\ 0 & s & -1 \\ 0 & -1 & 0\end{array}\right) H^{T}$, where $H=\left(v_{1}, v_{0}, v_{-1}\right)$ and $s$ is the parameter of the solution $v_{s, 0}$ of the Painlevé equation (17) determining the conformal factor $u$. It can be computed from $T$ by $s=\operatorname{tr} T$. If we normalize $v_{0}$ such that $\operatorname{det} H=-1$ and demand that $v_{n} \rightarrow \rho_{ \pm}$as $n \rightarrow \pm \infty$, then $v_{0}$ can be determined uniquely from the formula

$$
H\left(\begin{array}{ccc}
-1 & 0 & 0 \\
0 & s & -1 \\
0 & -1 & 0
\end{array}\right) H^{T}=\Phi_{-}(-\pi)\left(\begin{array}{ccc}
0 & 0 & -1 \\
0 & -1 & 0 \\
-1 & 0 & 3 \tilde{\alpha}_{a}
\end{array}\right) \Phi_{-}^{T}(0)
$$

In this section we described the boundaries of the self-associated cones corresponding to every solution $(u, U)$ of Wang's equation which has been obtained and analyzed in the previous sections. In the next section we summarize our findings in formal theorems.

\section{Results}

The self-associated cones can be grouped into three types according to Definition 6.1, which are described in the theorems below. Besides these, the only self-associated cones are the ellipsoidal cones, which form the $S L(3, \mathbb{R})$-orbit of the Lorentz cone $L_{3}$ described in Case 0 of Section 3.

Theorem 7.1. The $S L(3, \mathbb{R})$-orbits of self-associated cones of elliptic type are parameterized by a discrete parameter $k \in \mathbb{N}$ and a continuous parameter $R \in(0,+\infty]$. Every such cone possesses a subgroup of linear automorphisms which is isomorphic to the dihedral group $D_{k+3}$. This subgroup is generated by an automorphism $T$ with spectrum $\left\{1, e^{2 \pi i /(k+3)}, e^{-2 \pi i /(k+3)}\right\}$ and a reflection $\Sigma$. The cone possesses an interior ray which is left fixed by every automorphism in the subgroup.

The complete hyperbolic affine 2-sphere which is asymptotic to a cone of elliptic type corresponding to the parameters $(k, R)$ possesses an isothermal parametrization with domain $B_{R} \subset \mathbb{C}$, the open disc of radius $R$, and cubic differential $U=z^{k}$. The corresponding affine metric $h=e^{u}|d z|^{2}$ is given by the conformal factor $e^{u(z)}=\sqrt{\frac{k+3}{2}}|z|^{(k-1) / 2} v_{s_{k}, c}(t)$, where $t=\sqrt{\frac{32}{(k+3)^{3}}}|z|^{(k+3) / 2}, s_{k}=1+2 \cos \frac{2 \pi}{k+3}$, and $v_{s_{k}, c}$ is a solution of the Painlevé III equation (17). Here $c$ is a strictly monotonely decreasing function of $R$ such that $c=0$ for $R=+\infty$, and $c \rightarrow+\infty$ for $R \rightarrow 0$. For finite $R$ it is determined by the condition that $v_{s_{k}, c}$ is positive on $\left(0, t_{0}\right)$ and has a double pole at $t_{0}=\sqrt{\frac{32}{(k+3)^{3}}} R^{(k+3) / 2}$ with expansion (19).

For every $k \in \mathbb{N}$ the self-associated cones corresponding to the parameters $(k,+\infty)$ are linearly isomorphic to a cone over a regular $(k+3)$-gon.

The self-associated cones which correspond to the parameters $(k, R)$ for finite $R$ can be constructed as follows. Choose $W \in S L(3, \mathbb{R})$ arbitrarily and solve $O D E(12)$ with initial values $\left.\left(f_{0}^{\prime \prime}, f_{0}^{\prime}, f_{0}\right)\right|_{y=0}=-W$, where $e^{b}=\frac{R^{k+3}}{(k+3)^{3}}$ and $\tilde{\alpha}=\frac{\alpha t_{0}}{8}-\frac{1}{48}$. Then the solution $f_{0}: \mathbb{R} \rightarrow \mathbb{R}^{3}$ traces a closed analytic $2(k+3) \pi$ periodic curve in $\mathbb{R}^{3}$. The boundary of the cone is analytic at every non-zero point and can be obtained 

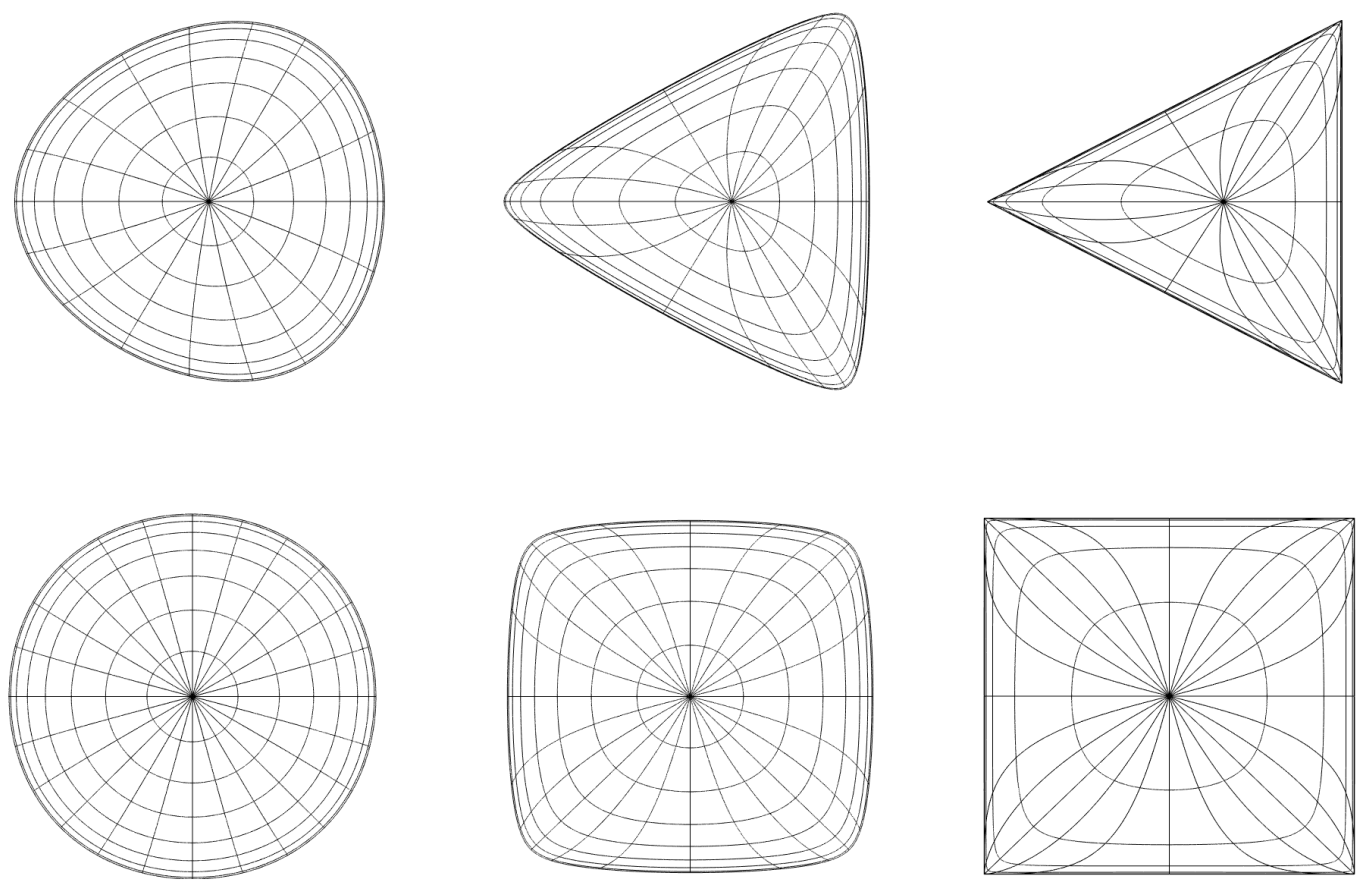

Figure 1: Compact affine sections of self-associated cones of elliptic type for $k=0,1$ and $R=1,2,4$ with uniform grid in polar coordinates of the domain $M=B_{R}$. The step size in the radial direction equals 1 , in the angular direction $\frac{\pi}{3}$.

as the conic hull of this curve. The $S L(3, \mathbb{R})$-orbit of self-associated cones corresponding to $(k, R)$ can be parameterized by the initial condition $W$.

In Fig. 1 we present compact affine sections of some self-associated cones of elliptic type. Since each interior ray intersects the affine sphere inscribed in the cone in exactly one point, we may project the isothermal coordinate $z \in B_{R}$ onto the interior of the affine section. A uniformly spaced polar coordinate grid on $B_{R}$ projects on the sections as shown in the figure.

Theorem 7.2. The $S L(3, \mathbb{R})$-orbits of self-associated cones of parabolic type are parameterized by a continuous parameter $b \in(-\infty,+\infty]$. Every such cone $K$ possesses a subgroup of linear automorphisms which is isomorphic to the infinite dihedral group $D_{\infty}$. This subgroup is generated by an automorphism $T$ with spectrum $\{1\}$ and eigenvalue of geometric multiplicity 1 , and a reflection $\Sigma$. There exists a boundary ray $\rho_{0}$ of $K$ which is left fixed by every automorphism in the subgroup.

The complete hyperbolic affine 2-sphere which is asymptotic to a cone of parabolic type corresponding to the parameter $b$ possesses an isothermal parametrization with domain $(-\infty, b)+i \mathbb{R}$ and cubic differential $U=e^{z}$. The corresponding affine metric $h=e^{u}|d z|^{2}$ is given by the conformal factor $e^{u(z)}=\frac{t v_{3, c}(t)}{8}$, where $t=\sqrt{32} e^{R e z / 2}$, and $v_{3, c}$ is a solution of the Painlevé III equation (17). Here c is a strictly monotonely decreasing function of $b$ such that $c=0$ for $b=+\infty$, and $c \rightarrow+\infty$ for $b \rightarrow-\infty$. For finite $b$ it is determined by the condition that $v_{3, c}$ is positive on $\left(0, t_{0}\right)$ and has a double pole at $t_{0}=\sqrt{32} e^{b / 2}$ with expansion (19).

Any cone corresponding to the parameter value $b=+\infty$ is linearly isomorphic to the cone given by the closed convex conic hull of the infinite set of vectors $v_{n}=\left(n^{2}, n, 1\right)^{T}, n \in \mathbb{Z}$.

The self-associated cones which correspond to a finite parameter $b$ can be constructed as follows. Choose $W \in S L(3, \mathbb{R})$ arbitrarily and solve the vector-valued $O D E(12)$ with initial values $\left.\left(f_{0}^{\prime \prime}, f_{0}^{\prime}, f_{0}\right)\right|_{y=0}=-W$, where $\tilde{\alpha}=\frac{\alpha e^{b / 2}}{\sqrt{2}}-\frac{1}{48}$. Then the solution $f_{0}: \mathbb{R} \rightarrow \mathbb{R}^{3}$ traces an analytic curve in $\mathbb{R}^{3}$. The conic hull of this curve is analytic at every non-zero point and meets itself at the ray $\rho_{0}$. The boundary of the cone can be obtained as the union of the conic hull of $f_{0}$ with the ray $\rho_{0}$. The $S L(3, \mathbb{R})$-orbit of self-associated cones corresponding to the parameter $b$ can be parameterized by the initial condition $W$.

In Fig. 2 we represent compact affine sections of self-associated cones of parabolic type for the parameter 

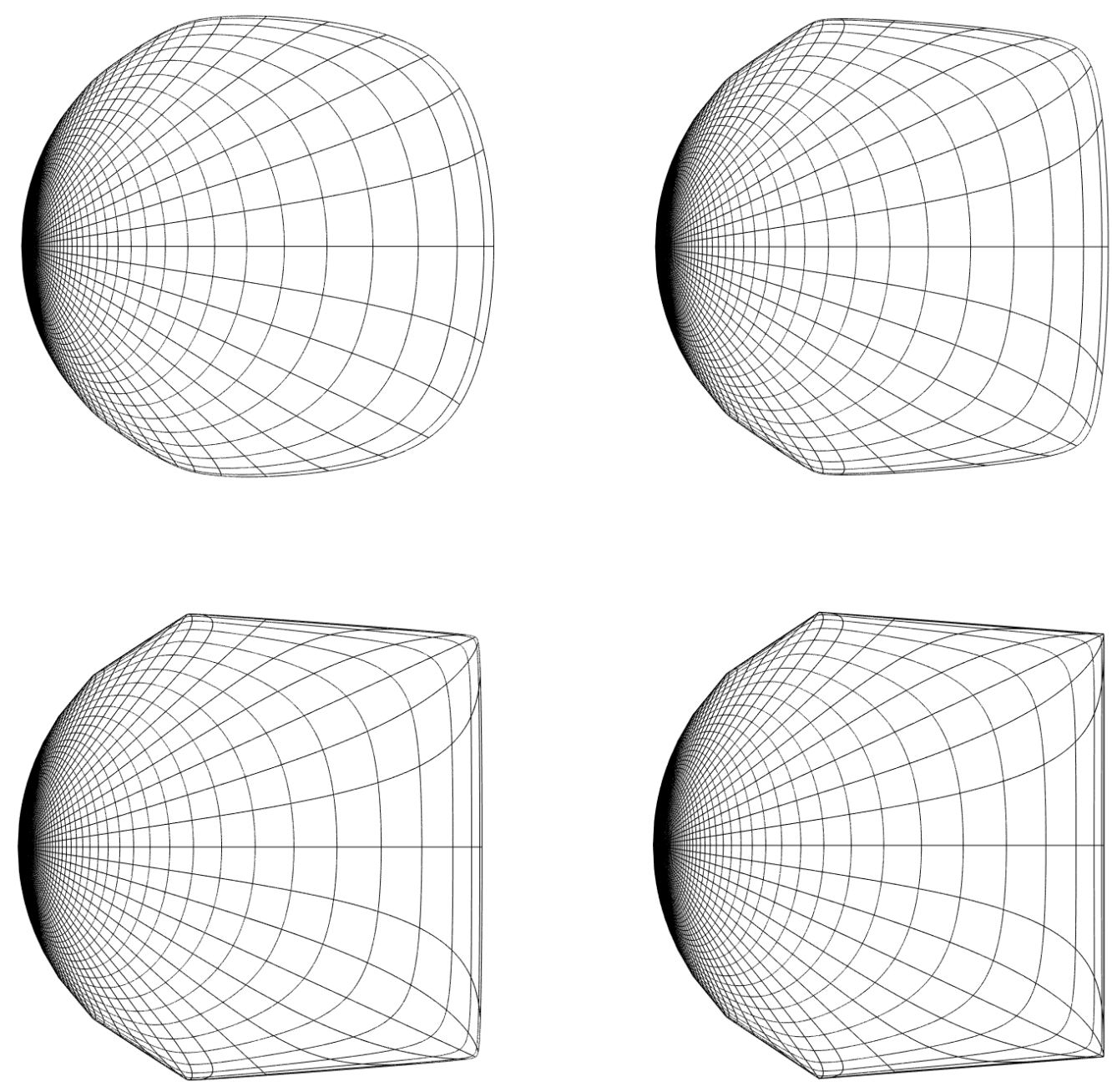

Figure 2: Compact affine sections of self-associated cones of parabolic type for $b=-2,-1,0,1$ with uniform grid in cartesian coordinates on the domain $M=(-\infty, b)+i \mathbb{R}$. The step size in the horizontal direction equals 1 , in the vertical direction $\frac{\pi}{3}$.

values $b=-2,-1,0,1$. As in Fig. 1, we project a uniformly spaced grid on the section.

Theorem 7.3. The $S L(3, \mathbb{R})$-orbits of self-associated cones of hyperbolic type are parameterized by two continuous parameters $-\infty<a<b \leq+\infty$. Every such cone $K$ possesses a subgroup of linear automorphisms which is isomorphic to the infinite dihedral group $D_{\infty}$. This subgroup is generated by an automorphism $T$ with spectrum $\left\{1, \lambda, \lambda^{-1}\right\}, \lambda>1$, and a reflection $\Sigma$. The cone possesses two boundary rays $\rho_{ \pm}$which are generated by eigenvectors of $T$ with eigenvalues $\lambda^{ \pm 1}$, respectively, and are mapped to each other by $\Sigma$.

The complete hyperbolic affine 2-sphere which is asymptotic to a cone of hyperbolic type corresponding to the parameters $(a, b)$ possesses an isothermal parametrization with domain $(a, b)+i \mathbb{R}$ and cubic differential $U=e^{z}$. The corresponding affine metric $h=e^{u}|d z|^{2}$ is given by the conformal factor $e^{u(z)}=\frac{t v(t)}{8}$, where $t=\sqrt{32} e^{R e z / 2}$, and $v$ is a solution of the Painlevé III equation (17). This solution is characterized by the condition that it is positive on $\left(t_{a}, t_{b}\right)$ with $t_{a}=\sqrt{32} e^{a / 2}, t_{b}=\sqrt{32} e^{b / 2}$, has a double pole at $t_{a}$ with expansion (19) featuring a constant $\alpha_{a}$, and for finite $b$ has a double pole at $t_{b}$ with expansion (19) featuring a constant $\alpha_{b}$. If $b=+\infty$, then $v$ is given by the solution $v_{s, 0}$, where $s$ is a strictly monotonely increasing function of $a$ such that $s \rightarrow 3$ for $a \rightarrow-\infty$, and $s \rightarrow+\infty$ for $a \rightarrow+\infty$. 
The boundary of $K$ is the closure of the union of two pieces which join at the rays $\rho_{ \pm}$.

The left piece can be constructed as follows. Choose $W \in S L(3, \mathbb{R})$ arbitrarily and solve the vector-valued $O D E$ (10) with initial values $\Phi_{-}(0)=\left.\left(f_{0}^{\prime \prime}, f_{0}^{\prime}, f_{0}\right)\right|_{y=0}=W$, where $\tilde{\alpha}=\frac{\alpha_{a} e^{a / 2}}{\sqrt{2}}-\frac{1}{48}$. Then the solution $f_{0}: \mathbb{R} \rightarrow \mathbb{R}^{3}$ traces an analytic curve in $\mathbb{R}^{3}$, whose conic hull is analytic at every non-zero point and yields the left piece of $\partial K$. The curve $f_{0}$ tends to $\rho_{ \pm}$for $y \rightarrow \pm \infty$, and $T=\left.\left(f_{0}^{\prime \prime}, f_{0}^{\prime}, f_{0}\right)\right|_{y=2 \pi} \cdot W^{-1}$.

The right piece of $\partial K$ can be constructed as follows.

Case $b<+\infty$ : Solve the vector-valued ODE (12) with initial values $\Phi_{+}(0)=\left.\left(f_{0}^{\prime \prime}, f_{0}^{\prime}, f_{0}\right)\right|_{y=0}=-W^{\prime}$, where $\tilde{\alpha}=\frac{\alpha_{b} e^{b / 2}}{\sqrt{2}}-\frac{1}{48}$, and $W^{\prime} \in S L(3, \mathbb{R})$ is determined by relations $(15)$ and the condition that $f_{0}(y)$ tends to $\rho_{ \pm}$as $y \rightarrow \pm \infty$, respectively. Then the solution $f_{0}: \mathbb{R} \rightarrow \mathbb{R}^{3}$ traces an analytic curve in $\mathbb{R}^{3}$, whose conic hull is analytic at every non-zero point and yields the right piece of $\partial K$.

Case $b=+\infty:$ Set $s=\operatorname{tr} T$ and let $v_{0} \in \mathbb{R}^{3}, v_{n}=T^{n} v_{0}, n \in \mathbb{Z}$, be non-zero vectors such that (16) holds with $H=\left(v_{1}, v_{0}, v_{-1}\right), \Sigma v_{n}=v_{1-n}$, and $v_{n} \rightarrow \rho_{ \pm}$for $n \rightarrow \pm \infty$, respectively. Then the right piece of $\partial K$ is given by an infinite chain of planar faces $F_{n}$ spanned by the vectors $v_{n}, v_{n+1}, n \in \mathbb{Z}$.

The $S L(3, \mathbb{R})$-orbit of self-associated cones corresponding to $(a, b)$ can be parameterized by the initial condition $W$.

In Fig. 3 we represent compact affine sections of self-associated cones of hyperbolic type for different parameter values $a, b$. As in the previous cases we project a uniformly spaced grid on the affine section.

\section{Open problems}

A cone $K \subset \mathbb{R}^{3}$ is self-associated if whenever there is another cone $\tilde{K} \subset \mathbb{R}^{3}$ such that the affine spheres which are asymptotic to the boundaries $\partial K, \partial \tilde{K}$ are isometric, the cones $K, \tilde{K}$ are linearly isomorphic. This characterization of self-associated cones can be generalized to higher dimensions and suggests the following problem.

Problem 1: For $n>3$, which cones $K \subset \mathbb{R}^{n}$ satisfy the following condition: whenever there exists another cone $\tilde{K} \subset \mathbb{R}^{n}$ such that the complete hyperbolic affine spheres with mean curvature $H=-1$ which are asymptotic to the boundaries $\partial K, \partial \tilde{K}$ are isometric, the cones $K, \tilde{K}$ are linearly isomorphic?

The affine spheres which are asymptotic to the boundary of self-associated cones possess a continuous group of isometries which multiply the cubic differential representing the cubic form by unimodular complex constants different from 1, and hence do not preserve the cubic form. We may then ask whether this is possible in higher dimensions.

Problem 2: For $n>3$, do there exist complete hyperbolic affine spheres $f: M \rightarrow \mathbb{R}^{n}$ with continuous groups of isometries which do not preserve the cubic form?

Any associated family of $S L(3, \mathbb{R})$-orbits of cones $K \subset \mathbb{R}^{3}$ admits a natural action of the circle group $S^{1}$, multiplying the holomorphic function $U$ representing the cubic differential by unimodular complex constants. For a self-associated cone its associated family consists of a single orbit. On the other hand, for a cone which is merely linearly isomorphic to its dual cone by a unimodular isomorphism we have that the action of the element $e^{i \pi} \in S^{1}$ leaves the orbits in the associated family fixed. This is a weaker condition than being self-associated. We may then consider the following intermediate notions.

Problem 3: Let $k \geq 3$ be an integer. Find cones $K \subset \mathbb{R}^{3}$ (other than the self-associated cones) such that the action of the element $e^{2 \pi i / k} \in S^{1}$ on its associated family of $S L(3, \mathbb{R})$-orbits of cones leaves the orbits fixed.

Affine spheres can be viewed as minimal Lagrangian manifolds in a certain para-Kähler space form [14]. The affine spheres corresponding to self-associated cones can hence be represented as minimal Lagrangian surfaces with a continuous symmetry group. Similar surfaces in the space $\mathbb{C P}^{2}$ have been considered in [11],[12] with loop group methods [10]. Surfaces with rotational and with translational symmetries have been distinguished and their loop group decompositions have been computed. Loop group methods are applicable to the case of definite affine 2 -spheres as well [22],[24].

Problem 4: Find the loop group decompositions of the affine spheres corresponding to the self-associated cones. 

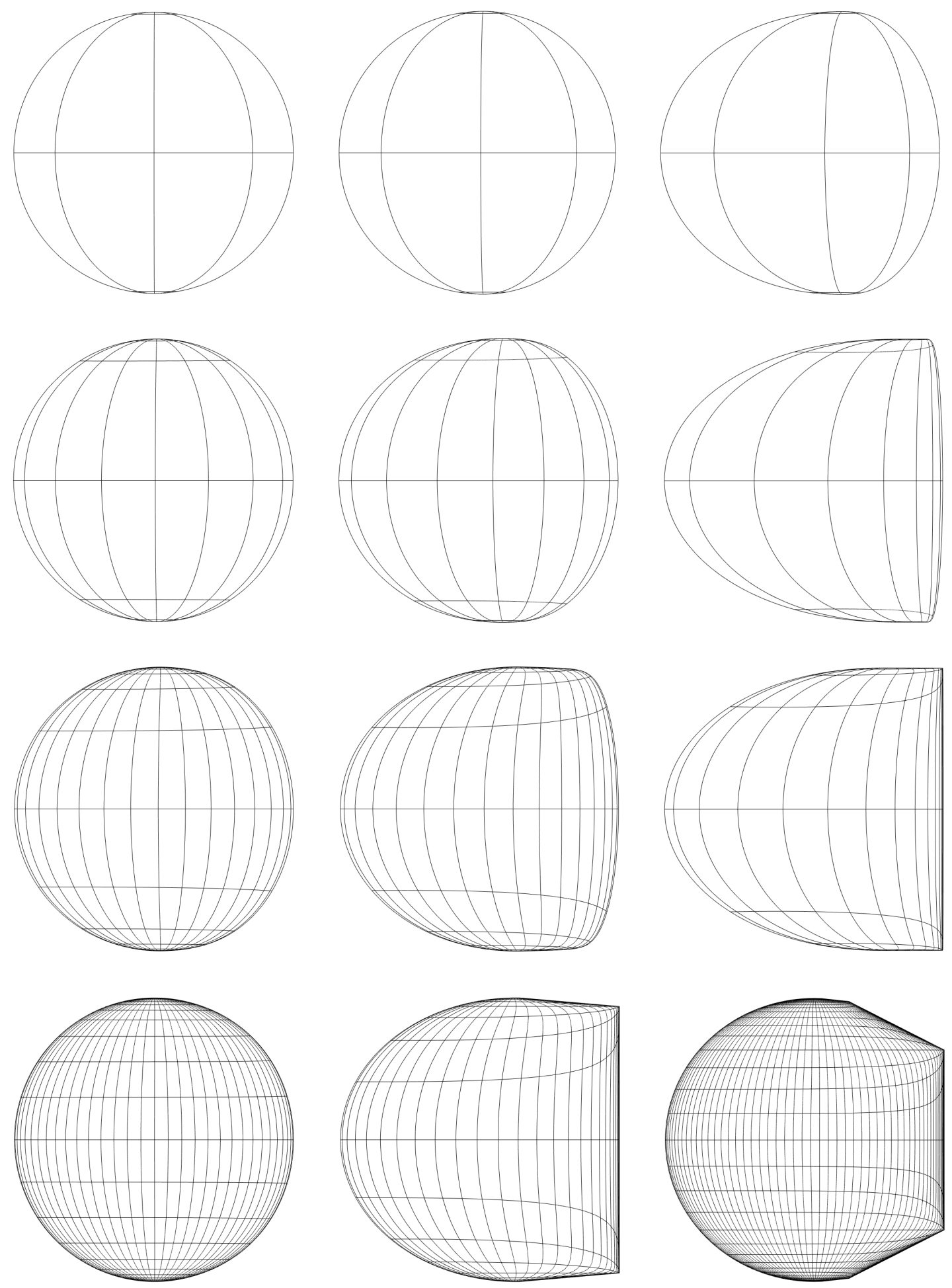

Figure 3: Compact affine sections of self-associated cones of hyperbolic type with uniform grid in cartesian coordinates on the domain $M=(a, b)+i \mathbb{R}$. The step size in the horizontal direction equals $\frac{1}{4}$, in the vertical direction $\frac{\pi}{4}$. The intervals $(a, b)$ are $(-3,2),(-1,0),(1,2)$ in the first row, $(-4,-2),(-2,0),(0,2)$ in the second row, $(-6,2),(-4,0),(-2,2)$ in the third row, and $(-12,-4),(-6,2),(-14,2)$ in the last row. 
Finally we shall formulate a conjecture on which property of a cone $K \subset \mathbb{R}^{3}$ is responsible for the conformal type of its corresponding affine sphere to be $\mathbb{C}$. The affine spheres corresponding to polyhedral cones have been shown to be conformal to $\mathbb{C}$ by Dumas and Wolf [13]. In this paper we have in addition constructed the self-associated cone of parabolic type corresponding to the parameter value $b=+\infty$ which has this property.

Conjecture: Let $K \subset \mathbb{R}^{3}$ be a cone. Then the following are equivalent:

(i) The complete hyperbolic affine spheres which are asymptotic to the boundary of $K$ have conformal type $\mathbb{C}$.

(ii) The union of the 2-dimensional faces of $K$ is dense in the boundary of $K$.

\section{Acknowledgements}

The author would like to thank Prof. Josef Dorfmeister for many inspiring discussions. In particular, he drew our attention to the fact that the reduction of Wang's equation is equivalent to the Painlevé III equation.

\section{A The degenerate Painlevé equation}

In this section we consider the Painlevé III equation

$$
t v v^{\prime \prime}=t\left(v^{\prime}\right)^{2}-v v^{\prime}+v^{3}-t
$$

with parameters $(\alpha, \beta, \gamma, \delta)=(1,0,0,-1)$, corresponding to the degenerate case $D_{7}$ in the classification of [30]. The ensemble of its solutions $v_{s, c}(t)$ can be parameterized by two complex parameters $s, c$ which encode monodromy data of an associated linear system of ODEs [16]. Equation (17) has no solution expressible in terms of algebraic and classical transcendental functions except $v_{0,0}(t)=t^{1 / 3}[29]$. Equation (17) has been studied in [17]. In particular, the asymptotics of the solutions in the neighbourhood of the singular points $t=0$ and $t=+\infty$ have been computed.

The real solutions which are pole-free and positive in a neighbourhood of $t=0$ on the positive real axis are parameterized by $(s, c) \in[-1,3] \times \mathbb{R}$, or equivalently $(\lambda, c) \in[0,1] \times \mathbb{R}$, where $\frac{s-1}{2}=\cos (\lambda \pi)$, and have the following asymptotics. For $s=3$ or $\lambda=0$

$$
v_{3, c}(t) t \sim \frac{2}{\left(\log t-3 \log 2+\frac{3}{2} \gamma+\frac{3}{4} c\right)^{2}},
$$

for $0<s<3$ or $\lambda \in\left(0, \frac{2}{3}\right)$

$$
v_{s, c}(t) t \sim \frac{2 \lambda^{2}}{\sinh ^{2}\left(\lambda \log t-3 \lambda \log 2+\frac{1}{2} \log \frac{\Gamma\left(1-\frac{\lambda}{2}\right) \Gamma(1-\lambda)}{\Gamma\left(1+\frac{\lambda}{2}\right) \Gamma(1+\lambda)}+\frac{3 \lambda}{4} c\right)},
$$

for $s=0$ or $\lambda=\frac{2}{3}$

$$
v_{0, c}(t) t^{-1 / 3} \sim e^{c}\left(1+\frac{9\left(e^{c}-e^{-2 c}\right)}{16} t^{4 / 3}\right)
$$

for $-1<s<0$ or $\lambda \in\left(\frac{2}{3}, 1\right)$

$$
v_{s, c}(t) t^{-1} \sim \frac{1}{2-2 \lambda} \sinh \left(-(2-2 \lambda) \log t+(6-6 \lambda) \log 2-\log \frac{-\Gamma\left(\frac{\lambda}{2}\right) \Gamma(-1+\lambda)}{\Gamma\left(1-\frac{\lambda}{2}\right) \Gamma(1-\lambda)}+3(1-\lambda) c\right),
$$

for $s=-1$ or $\lambda=1$

$$
v_{-1, c}(t) t^{-1} \sim-\log t+2 \log 2-\frac{3}{2} \gamma+\frac{3}{2} c,
$$

where $\gamma$ is the Euler-Mascheroni constant ${ }^{1}$.

\footnotetext{
${ }^{1}$ In the original paper [17] a factor of 2 is missing in the last expression.
} 
The real solutions which are pole-free and positive in a neighbourhood of $t=+\infty$ on the positive real axis are parameterized by $s \in \mathbb{R}$, the second parameter $c$ being zero, and have the asymptotics

$$
v_{s, 0}(t) t^{-1 / 3}-1 \sim 3^{-1 / 4} \pi^{-1 / 2} s t^{-1 / 3} e^{-3 \sqrt{3} t^{2 / 3} / 2} .
$$

The solutions $v_{s, 0}$ with $s \in[-1,3]$ appear in both lists, and their asymptotics both at $t=0$ and $t=+\infty$ are known.

For studying positive solutions $v(t)$ of (17) it is convenient to make the substitution $\tau=\log t, g=$ $\log v-\frac{1}{3} \log t$. Then (17) is equivalent to the second-order ODE

$$
\frac{d^{2} g}{d \tau^{2}}=e^{4 \tau / 3}\left(e^{g}-e^{-2 g}\right)
$$

on the function $g(\tau)$. By virtue of the strict monotonicity of the function $e^{g}-e^{-2 g}$ we have $g^{\prime \prime}>\tilde{g}^{\prime \prime}$ for two solutions $g, \tilde{g}$ whenever $g>\tilde{g}$.

We have the following monotonicity result.

Lemma A.1. Let $t_{b} \geq 0$ and $s<s^{\prime}$ be real numbers such that the solutions $v_{s, 0}, v_{s^{\prime}, 0}$ of (17) are positive on the interval $\left(t_{b},+\infty\right)$. Let $g_{s}, g_{s^{\prime}}$ be the corresponding solutions of (18). Then $g_{s^{\prime}}>g_{s}, g_{s^{\prime}}^{\prime}<g_{s}^{\prime}$ on $\left(\log t_{b},+\infty\right)$ and $v_{s, 0}<v_{s^{\prime}, 0}$ on $\left(t_{b},+\infty\right)$.

Proof. The asymptotics of $v_{s, 0}$ for $t \rightarrow+\infty$ yields

$$
g_{s}(\tau) \sim 3^{-1 / 4} \pi^{-1 / 2} s e^{-\tau / 3-3 \sqrt{3} \exp (2 \tau / 3) / 2}
$$

as $\tau \rightarrow+\infty$. Define the function $\delta=g_{s^{\prime}}-g_{s}$ on $\left(\log t_{b},+\infty\right)$. We get

$$
\delta(\tau) \sim 3^{-1 / 4} \pi^{-1 / 2}\left(s^{\prime}-s\right) e^{-\tau / 3-3 \sqrt{3} \exp (2 \tau / 3) / 2}
$$

as $\tau \rightarrow+\infty$. Moreover, $\delta^{\prime \prime}>0$ for all $\tau$ such that $\delta(\tau)>0$.

Now we have $\delta(\tau)>0$ for large enough $\tau$, and $\lim _{\tau \rightarrow+\infty} \delta(\tau)=0$. Hence there exists a sequence $\tau_{k} \rightarrow+\infty$ such that $\delta\left(\tau_{k}\right)>0, \delta^{\prime}\left(\tau_{k}\right) \leq 0$ for all $k$. But then $\delta^{\prime \prime}(\tau)>0, \delta^{\prime}(\tau)<0, \delta(\tau)>0$ for all $\tau<\tau_{k}$, and hence for all $\tau \in\left(\log t_{b},+\infty\right)$. The claim of the lemma now easily follows.

By the Painlevé property the solutions of (17) can be extended to meromorphic functions on the universal cover of $\mathbb{C} \backslash\{0\}$. If $t=t_{0} \neq 0$ is a singularity of the solution, then it must be a double pole with expansion

$$
v(t)=\frac{2 t_{0}}{\left(t-t_{0}\right)^{2}}+\alpha-\frac{\alpha}{t_{0}}\left(t-t_{0}\right)+\frac{3 \alpha^{2} t_{0}+9 \alpha}{10 t_{0}^{2}}\left(t-t_{0}\right)^{2}-\frac{3 \alpha^{2} t_{0}+4 \alpha}{5 t_{0}^{3}}\left(t-t_{0}\right)^{3}+\ldots
$$

The family $\left(v_{\alpha}\right)_{\alpha \in \mathbb{R}}$ of solutions having a double pole at $t_{0}$ satisfies a similar monotonicity result.

Lemma A.2. Let $t_{0}>0, \alpha<\alpha^{\prime}$ be real numbers, and let $v_{\alpha}, v_{\alpha^{\prime}}$ be the solutions of (17) with the corresponding expansions (19). Suppose that $v_{\alpha}, v_{\alpha^{\prime}}$ are positive on the interval $\left(t_{0}, T\right)$ for some $T \in\left(t_{0},+\infty\right]$, and let $g_{\alpha}, g_{\alpha^{\prime}}$ be the corresponding solutions of (18) on $\left(\tau_{0}, \log T\right)$, where $\tau_{0}=\log t_{0}$. Then we have $g_{\alpha}<g_{\alpha^{\prime}}$, $g_{\alpha}^{\prime}<g_{\alpha^{\prime}}^{\prime}$ on $\left(\tau_{0}, \log T\right)$, and $v_{\alpha}<v_{\alpha^{\prime}}$ on $\left(t_{0}, T\right)$.

Proof. Set $\delta=g_{\alpha^{\prime}}-g_{\alpha}$. Since the ratio $\frac{v_{\alpha^{\prime}}}{v_{\alpha}}$ is analytic and non-zero in the neighbourhood of $t_{0}$, its logarithm is also analytic and by virtue of (19) has the expansion

$$
\delta\left(\tau_{0}+d\right)=\frac{\alpha^{\prime}-\alpha}{2 t_{0}} d^{2}-\frac{\alpha^{\prime}-\alpha}{2 t_{0}^{2}} d^{3}+\frac{t_{0}\left(\left(\alpha^{\prime}\right)^{2}-\alpha^{2}\right)+18\left(\alpha^{\prime}-\alpha\right)}{40 t_{0}^{3}} d^{4}-\frac{t_{0}\left(\left(\alpha^{\prime}\right)^{2}-\alpha^{2}\right)+8\left(\alpha^{\prime}-\alpha\right)}{20 t_{0}^{4}} d^{5}+\ldots
$$

It follows that for $\tau>\tau_{0}$ close enough to $\tau_{0}$ we have $\delta(\tau)>0$ and $\delta^{\prime}(\tau)>0$. But $\delta^{\prime \prime}>0$ as long as $\delta>0$, hence these relations are valid on the whole interval $\left(\tau_{0}, \log T\right)$. The claim of the lemma now easily follows.

Corollary A.3. Let $t_{0}>0, \alpha<0$. Then the solution $v_{\alpha}$ of (17) with expansion (19) obeys $v_{\alpha}(t)<\frac{2 t_{0}}{\left(t-t_{0}\right)^{2}}$ for all $t$ such that $v_{\alpha}$ is positive on $\left(t_{0}, t\right)$. 
Proof. Let $g_{\alpha}(\tau)$ be the solution of (18) corresponding to $v_{\alpha}$, and set $\tau_{0}=\log t_{0}, g_{0}(\tau)=\log 2+\tau_{0}-$ $2 \log \left(e^{\tau}-e^{\tau_{0}}\right)-\frac{1}{3} \tau, \delta=g_{0}-g_{\alpha}=-\log \frac{\left(t-t_{0}\right)^{2} v_{\alpha}(t)}{2 t_{0}}$. By (19) the function $\delta$ is analytic at $\tau=\tau_{0}$ with expansion $\delta(\tau) \sim-\frac{\alpha e^{\tau_{0}}}{2}\left(\tau-\tau_{0}\right)^{2}+\ldots$ For $\tau>\tau_{0}$ close enough to $\tau_{0}$ we then have $\delta(\tau)>0, \delta^{\prime}(\tau)>0$. Moreover, as long as $\delta(\tau)>0$ we have

$$
\delta^{\prime \prime}(\tau)=\frac{2 e^{\tau_{0}} e^{\tau}}{\left(e^{\tau}-e^{\tau_{0}}\right)^{2}}-e^{4 \tau / 3}\left(e^{g_{\alpha}}-e^{-2 g_{\alpha}}\right)>e^{4 \tau / 3}\left(e^{g_{0}}-e^{g_{\alpha}}\right)>0 .
$$

Hence $\delta(\tau)>0$ as long as this function is defined, and the claim of the corollary easily follows.

The monotonicity lemmas have the following consequence.

Corollary A.4. Let $t_{0}>0$. Then there exists at most one value $s \in \mathbb{R}$ such that the solution $v_{s, 0}$ of (17) is positive on $\left(t_{0},+\infty\right)$ and has a pole at $t=t_{0}$.

Proof. Suppose there exist two different values $s<s^{\prime}$ satisfying the conditions in the Corollary. Let $g_{s}, g_{s^{\prime}}$ be the corresponding solutions of (18). Then by Lemma A.1 we have $g_{s^{\prime}}>g_{s}, g_{s^{\prime}}^{\prime}<g_{s}^{\prime}$ on $\left(t_{0},+\infty\right)$. However, by Lemma A.2 these two inequalities are incompatible. This concludes the proof.

Finally, we shall estimate the error in the asymptotics of the solution $v_{3, c}$ provided in [17]. For given $c \in \mathbb{R}$, set for brevity $c^{\prime}=3 \log 2-\frac{3}{2} \gamma-\frac{3}{4} c$.

Lemma A.5. The asymptotics of the solution $v_{3, c}$ for $t \rightarrow 0$ is given by $\frac{2 t^{-1}}{\left(-\log t+c^{\prime}\right)^{2}}-\frac{t^{3}\left(-\log t+c^{\prime}+1\right)^{2}}{32}$. The asymptotics of its derivative is given by $\frac{d v_{3, c}}{d t} \sim-\frac{2 t^{-2}}{\left(-\log t+c^{\prime}\right)^{2}}+\frac{4 t^{-2}}{\left(-\log t+c^{\prime}\right)^{3}}-\frac{3 t^{2}\left(-\log t+c^{\prime}+\frac{2}{3}\right)^{2}}{32}$.

Proof. The solution $g(\tau)$ of (18) which corresponds to $v_{3, c}$ has asymptotics

$$
g(\tau) \sim \log 2-\frac{4}{3} \tau-2 \log \left(-\tau+c^{\prime}\right)=: \tilde{g}(\tau) .
$$

Note that the function $\tilde{g}$ satisfies the ODE $\tilde{g}^{\prime \prime}=e^{4 \tau / 3} e^{\tilde{g}}$, and hence $\delta=\tilde{g}-g$ satisfies

$$
\delta^{\prime \prime}=e^{4 \tau / 3}\left(e^{\tilde{g}}-e^{g}+e^{-2 g}\right)=2\left(-\tau+c^{\prime}\right)^{-2}\left(1-e^{-\delta}\right)+\mu(\tau),
$$

where $\mu(\tau)=e^{4 \tau / 3} e^{-2 g} \sim \frac{1}{4} e^{4 \tau}\left(-\tau+c^{\prime}\right)^{4}$.

Define the functions $\underline{\mu}(\tau)=\int_{-\infty}^{\tau} \int_{-\infty}^{s} \mu(r) d r d s$ and $\bar{\mu}(\tau)=\frac{1}{-\tau+c^{\prime}} \int_{-\infty}^{\tau}\left(s-c^{\prime}\right)^{2} \int_{-\infty}^{s} \frac{\mu(r)}{-r+c^{\prime}} d r d s$. Note that $\bar{\mu}^{\prime \prime}=\frac{2 \bar{\mu}}{\left(\tau-c^{\prime}\right)^{2}}+\mu>\frac{2}{\left(\tau-c^{\prime}\right)^{2}}\left(1-e^{-\bar{\mu}}\right)+\mu$. Note that both $\underline{\mu}$ and $\bar{\mu}$ have the same asymptotics $\frac{e^{4 \tau}\left(-\tau+c^{\prime}+\frac{1}{2}\right)^{4}}{64}$ as $\tau \rightarrow-\infty$. We shall now show that $\underline{\mu}<\delta<\bar{\mu}$.

Let $\tau_{0}<c^{\prime}$ be such that $g$ and hence also $\mu$ and $\delta$ are defined on $\left(-\infty, \tau_{0}\right]$. Consider the integral operator $\mathcal{I}$ taking a smooth function $f:\left(-\infty, \tau_{0}\right] \rightarrow \mathbb{R}$ to $[\mathcal{I} f](\tau)=\int_{-\infty}^{\tau} \int_{-\infty}^{s} \frac{2}{\left(-r+c^{\prime}\right)^{2}}\left(1-e^{-f(r)}\right)+\mu(r) d r d s$. If $f$ is such that $0 \leq f(\tau) \leq \bar{\mu}(\tau)$ for all $\tau \in\left(-\infty, \tau_{0}\right]$, then

$$
0 \leq[\mathcal{I} f](\tau) \leq \int_{-\infty}^{\tau} \int_{-\infty}^{s} \frac{2}{\left(-r+c^{\prime}\right)^{2}}\left(1-e^{-\bar{\mu}(r)}\right)+\mu(r) d r d s<\int_{-\infty}^{\tau} \int_{-\infty}^{s} \bar{\mu}^{\prime \prime}(r) d r d s=\bar{\mu}(\tau), \quad \tau \leq \tau_{0}
$$

Hence $\mathcal{I}$ is well-defined for every such function $f$. Clearly if $f<\tilde{f}$ everywhere on $\left(-\infty, \tau_{0}\right]$, then also $\mathcal{I} f<\mathcal{I} \tilde{f}$ everywhere on this interval.

Define now $\delta_{0} \equiv 0$ and recursively $\delta_{k+1}=\mathcal{I} \delta_{k}, k \geq 0$. Then $0=\delta_{0}<\delta_{1}=\mu$, and hence the sequence $\delta_{k}(\tau)$ is strictly increasing for every $\tau \leq \tau_{0}$. On the other hand, this sequence is upper bounded by $\bar{\mu}(\tau)$. Hence $\delta_{k}$ converges point-wise to some limit function $\delta^{*}$. This function is a fixed point of the operator $\mathcal{I}$ and hence smooth and a solution of ODE (20). Thus it coincides with the sought function $\delta$. By construction we obtain the desired bounds $\underline{\mu}<\delta<\bar{\mu}$ and therefore the asymptotics $\delta \sim \frac{e^{4 \tau}\left(-\tau+c^{\prime}+\frac{1}{2}\right)^{4}}{64}$.

Switching back to the solution $v(t)$ of $(17)$ we get

$$
v_{3, c}(t)=t^{1 / 3} e^{\tilde{g}-\delta} \sim \frac{2 t^{-1}}{\left(-\log t+c^{\prime}\right)^{2}}\left(1-\frac{t^{4}\left(-\log t+c^{\prime}+\frac{1}{2}\right)^{4}}{64}\right)
$$


which yields the desired error term for $v_{3, c}$. that

Further, by (20) we have $\delta^{\prime \prime} \sim \frac{e^{4 \tau}\left(-\tau+c^{\prime}\right)^{4}}{4}$ and hence $\delta^{\prime}(\tau)=\int_{-\infty}^{\tau} \delta^{\prime \prime}(s) d s \sim \frac{e^{4 \tau}\left(-\tau+c^{\prime}+\frac{1}{4}\right)^{4}}{16}$. It follows

$$
g^{\prime}(\tau)=\tilde{g}^{\prime}-\delta^{\prime} \sim-\frac{4}{3}+\frac{2}{-\tau+c^{\prime}}-\frac{e^{4 \tau}\left(-\tau+c^{\prime}+\frac{1}{4}\right)^{4}}{16} .
$$

Therefore by virtue of $\frac{d \tau}{d t}=t^{-1}$ we have

$$
\begin{aligned}
\frac{d v_{3, c}}{d t} & =t^{-2 / 3} e^{\tilde{g}-\delta}\left(g^{\prime}+\frac{1}{3}\right) \\
& \sim \frac{2 t^{-2}}{\left(-\log t+c^{\prime}\right)^{2}}\left(1-\frac{t^{4}\left(-\log t+c^{\prime}+\frac{1}{2}\right)^{4}}{64}\right)\left(-1+\frac{2}{-\log t+c^{\prime}}-\frac{t^{4}\left(-\log t+c^{\prime}+\frac{1}{4}\right)^{4}}{16}\right),
\end{aligned}
$$

which after a little calculus yields the desired error term.

\section{References}

[1] Yves Benoist and Dominique Hulin. Cubic differentials and finite volume convex projective surfaces. Geom. Topol., 17(1):595-620, 2013.

[2] Yves Benoist and Dominique Hulin. Cubic differentials and hyperbolic convex sets. J. Differ. Geom., 98(1):1-19, 2014.

[3] Alexander I. Bobenko and Ulrich Eitner. Painlevé equations in the differential geometry of surfaces, volume 1753 of Lecture Notes in Mathematics. Springer, Berlin, 2000.

[4] Alexander I. Bobenko, Ulrich Eitner, and Alexander V. Kitaev. Surfaces with harmonic inverse mean curvature and Painlevé equations. Geometriae Dedicata, 68:187-227, 1997.

[5] Alexander I. Bobenko and Alexander Its. The Painlevé III equation and the Iwasawa decomposition. Manuscripta Math., 87:369-377, 1995.

[6] Alexander I. Bobenko and Alexander V. Kitaev. On asymptotic cones of surfaces with constant curvature and the third Painlevé equation. Manuscripta Math., 97:489-516, 1998.

[7] Eugenio Calabi. Complete affine hyperspheres I. In Symposia Mathematica Vol. 10, pages 19-38. Istituto Nazionale di Alta Matematica, Acad. Press, 1972.

[8] Shiu Yuen Cheng and Shing-Tung Yau. On the regularity of the Monge-Ampère equation $\operatorname{det}\left(\partial^{2} u / \partial x_{i} \partial x_{j}\right)=f(x, u)$. Comm. Pure Appl. Math., 30:41-68, 1977.

[9] Shiu Yuen Cheng and Shing-Tung Yau. Complete affine hypersurfaces, Part I. The completeness of affine metrics. Comm. Pure Appl. Math., 39:839-866, 1986.

[10] Josef F. Dorfmeister and Ulrich Eitner. Weierstrass type representation of affine spheres. Abh. Math. Sem. Univ. Hambg., 71:225-250, 2001.

[11] Josef F. Dorfmeister and Hui Ma. Explicit expressions for the Iwasawa factors, the metric and the monodromy matrices for minimal Lagrangian surfaces in $\mathbb{C P}^{2}$. In Dynamical Systems, Number Theory and Applications, chapter 2, pages 19-47. World Scientific, 2016.

[12] Josef F. Dorfmeister and Hui Ma. A new look at equivariant minimal Lagrangian surfaces in $\mathbb{C P}^{2}$. In Akito Futaki, Reiko Miyaoka, Zizhou Tang, and Weiping Zhang, editors, Geometry and Topology of Manifolds: 10th China-Japan Conference 2014, volume 154 of Springer Proceedings in Mathematics and Statistics, pages 97-126. Springer, Tokyo, 2016.

[13] David Dumas and Michael Wolf. Polynomial cubic differentials and convex polygons in the projective plane. Geom. Funct. Anal., 25:1734-1798, 2015. 
[14] Roland Hildebrand. The cross-ratio manifold: a model of centro-affine geometry. Int. Electron. J. Geom., $4(2): 32-62,2011$.

[15] Roland Hildebrand. Analytic formulas for complete hyperbolic affine spheres. Contributions to Algebra and Geometry, 55(2):497-520, 2014.

[16] Alexander R. Its and Victor Yu. Novokshenov. The isomonodromic deformation method in the theory of Painlevé equations, volume 1191 of Lecture Notes in Mathematics. Springer, Berlin, 1986.

[17] Alexander V. Kitaev. The method of isomonodromic deformations for the degenerate third Painlevé equation. Zap. Nauchn. Sem. LOMI, 161:45-53, 1987.

[18] François Labourie. Flat projective structures on surfaces and cubic holomorphic differentials. Pure Appl. Math. Q., 3(4):1057-1099, 2007.

[19] An-Min Li. Calabi conjecture on hyperbolic affine hyperspheres. Math. Z., 203:483-491, 1990.

[20] An-Min Li. Calabi conjecture on hyperbolic affine hyperspheres (2). Math. Ann., 293:485-493, 1992.

[21] An-Min Li, Udo Simon, and Guosong Zhao. Global Affine Differential Geometry of Hypersurfaces, volume 11 of De Gruyter Expositions in Mathematics. Walter de Gruyter, 1993.

[22] Mingheng Liang and Qingchun Ji. Definite affine spheres and loop groups. J. Geom. Phys., 60:782-790, 2010.

[23] Zhicheng Lin and Erxiao Wang. The associated families of semi-homogeneous complete hyperbolic affine spheres. Acta Math. Sci. (English Ed.), 36(3):765-781, 2016.

[24] Zhicheng Lin, Gang Wang, and Erxiao Wang. Dressing actions on proper definite affine spheres. Asian J. Math., 21(2):363-390, 2017.

[25] John C. Loftin. Applications of affine differential geometry to $\mathbb{R} \mathbb{P}(2)$ surfaces. PhD thesis, Harvard University, Cambridge, MA, 1999.

[26] John C. Loftin. Affine spheres and convex $\mathbb{R}^{n}$-manifolds. Amer. J. Math., 123(2):255-275, 2001.

[27] John C. Loftin. The compactification of the moduli space of convex $\mathbb{R P}^{2}$ surfaces. I. J. Differ. Geom., 68:223-276, 2004.

[28] Katsumi Nomizu and Takeshi Sasaki. Affine Differential Geometry: Geometry of Affine Immersions, volume 111 of Cambridge Tracts in Mathematics. Cambridge University Press, Cambridge, 1994.

[29] Yousuke Ohyama, Hiroyuki Kawamuko, Hidetaka Sakai, and Kazuo Okamoto. Studies on the Painlevé equations, V, Third Painlevé equations of special type $P_{I I I}\left(D_{7}\right)$ and $P_{I I I}\left(D_{8}\right)$. J. Math. Sci. Univ. Tokyo, 13:145-204, 2006.

[30] H. Sakai. Rational surfaces associated with affine root systems and geometry of the Painlevé equations. Comm. Math. Phys., 220:165-229, 2001.

[31] Takeshi Sasaki. Hyperbolic affine hyperspheres. Nagoya Math. J., 77:107-123, 1980.

[32] Udo Simon and Chang-Ping Wang. Local theory of affine 2-spheres. In Differential Geometry: Riemannian geometry, volume 54-3 of Proceedings of Symposia in Pure Mathematics, pages 585-598. American Mathematical Society, Los Angeles, 1993.

[33] Chang-Ping Wang. Some examples of complete hyperbolic affine 2-spheres in $\mathbb{R}^{3}$. In Global Differential Geometry and Global Analysis, volume 1481 of Lecture Notes in Mathematics, pages 272-280. Springer, 1991. 